\title{
Homotopy and dynamics for homeomorphisms of solenoids and Knaster continua
}

\author{
by \\ Jarosław Kwapisz (Bozeman, MT)
}

\begin{abstract}
We describe the homotopy classes of self-homeomorphisms of solenoids and Knaster continua. In particular, we demonstrate that homeomorphisms within one homotopy class have the same (explicitly given) topological entropy and that they are actually semi-conjugate to an algebraic homeomorphism in the case when the entropy is positive.
\end{abstract}

1. Introduction. A solenoid goes back to $[20,5]$ and is an indecomposable continuum that can be visualized as intersection of a nested sequence of progressively thinner solid tori that are each wrapped into the previous one a number of times as suggested by Figure 1.1. Any radial cross section of a solenoid is a Cantor set each point of which belongs to a densely immersed line, called a composant. The wrapping numbers may vary from one torus to another; we shall record their sequence by $\mathcal{P}=\left\{p_{1}, p_{2}, \ldots\right\}$, and we shall refer to the associated solenoid as the $\mathcal{P}$-adic solenoid, denoted by $\mathcal{S}_{\mathcal{P}}$. No generality is lost in assuming that all $p_{i}$ 's are prime.

For the sequence $\mathcal{P}$, we also have the associated $\mathcal{P}$-adic Knaster continuum $\mathcal{K}_{\mathcal{P}}$ that is the intersection of a nested sequence of disks each traversing the previous one in a snake-like fashion a number of times indicated by the corresponding term in $\mathcal{P}$ (see Figure 1.2). $\mathcal{K}_{\mathcal{P}}$ is related to $\mathcal{S}_{\mathcal{P}}$ by a $2-1$ branched cover $\mathcal{S}_{\mathcal{P}} \rightarrow \mathcal{K}_{\mathcal{P}}$ (see Section 9 and [3]).

In dynamics, which serves as our main motivation, one usually encounters $\mathcal{S}_{\mathcal{P}}$ and $\mathcal{K}_{\mathcal{P}}$ for periodic sequences $\mathcal{P}, \mathcal{P}=\{n, n, \ldots\}$. Particularly, for $n=2$ we get two classical attractors: Smale's Solenoid and Smale's Horseshoe (see e.g. [11]). These are basic examples among a bewildering variety

2000 Mathematics Subject Classification: Primary 37B40, 37B45; Secondary 28D20, $54 \mathrm{H} 20$.

This work has been partially supported by NSF EPSCoR grant MONTS-190729 and NSF grant DMS-9970725. 

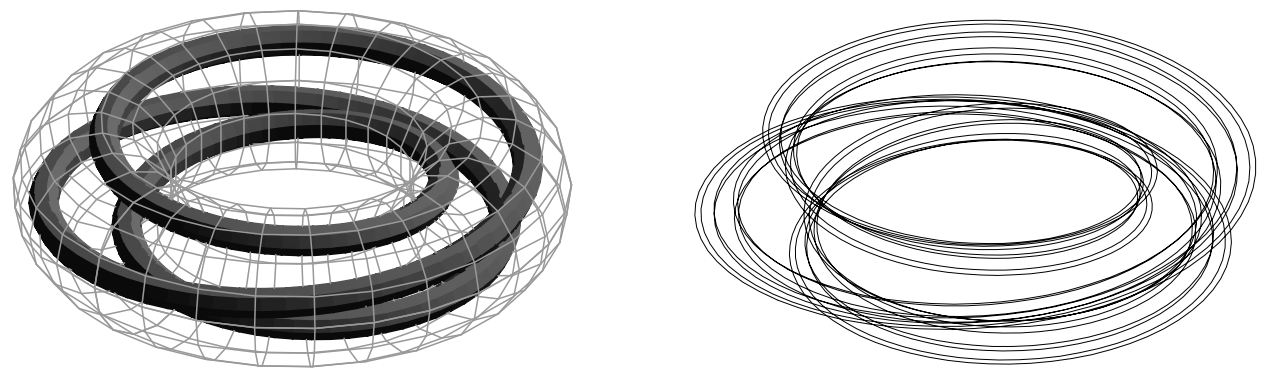

Fig. 1.1. Two tori-one wrapped into another 3 times - and an approximation to the $(3,2,2, \ldots)$-adic solenoid
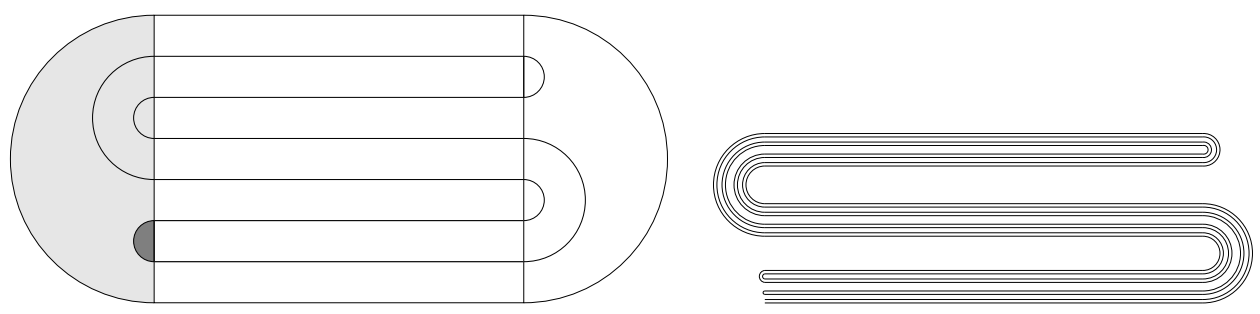

Fig. 1.2. Two embedded disks and an approximation to the $(3,2,2, \ldots)$-adic Knaster continuum

of complicated continua that are observed in chaotic systems. One naturally wonders to what extent the rich structure of such sets determines the underlying dynamics. We solve this problem in the simplest setting of $\mathcal{S}_{\mathcal{P}}$ and $\mathcal{K}_{\mathcal{P}}$ by classifying all their homeomorphisms. Let us describe our results for $\mathcal{S}_{\mathcal{P}}$ now; analogous theorems hold for $\mathcal{K}_{\mathcal{P}}$ (see Section 9).

$\mathcal{S}_{\mathcal{P}}$ has the structure of a topological group (see Section 2 or [10]). Its translations roughly turn $\mathcal{S}_{\mathcal{P}}$ axially and may fix or permute its composants. Besides translations and the involution $r: z \mapsto z^{-1}$, the simplest maps of $\mathcal{S}_{\mathcal{P}}$ are the Frobenius homomorphisms $g_{b}: \mathcal{S}_{\mathcal{P}} \rightarrow \mathcal{S}_{\mathcal{P}}, z \mapsto z^{b}$, where $b \in \mathbb{N}$. (The action of $g_{b}$ roughly wraps $\mathcal{S}_{\mathcal{P}}$ longitudinally $b$ times onto itself.) We shall prove that $g_{b}$ is a homeomorphism iff $b$ is $\mathcal{P}$-recurrent (i.e. every prime dividing $b$ repeats infinitely many times in $\mathcal{P}$ ). In that case, we can also form the maps $g_{a / b}:=g_{b}^{-1} \circ g_{a}$. The compositions $s \circ g_{a / b}$ and $r \circ s \circ g_{a / b}$ where $s$ is a translation and $a, b \in \mathbb{N}$ are co-prime and $\mathcal{P}$-recurrent form a group of affine homeomorphisms of $\mathcal{S}_{\mathcal{P}}$.

Theorem $1\left({ }^{1}\right)$. Suppose that $\mathcal{P}$ is an infinite sequence of prime numbers and $f: \mathcal{S}_{\mathcal{P}} \rightarrow \mathcal{S}_{\mathcal{P}}$ is a homeomorphism of the $\mathcal{P}$-adic solenoid. Then there are unique $\mathcal{P}$-recurrent and co-prime natural numbers $a, b \in \mathbb{N}$ such that $f$ is isotopic to the affine map $g=s \circ g_{a / b}$ or $g=r \circ s \circ g_{a / b}$.

$\left({ }^{1}\right)$ This result is hardly new; see the comments at the end of this introduction. 
We note that $g$ is determined uniquely up to a translation that fixes the composants of $\mathcal{S}_{\mathcal{P}}$ so that there are actually uncountably many homotopy classes for homeomorphisms of $\mathcal{S}_{\mathcal{P}}$; each class is determined by the winding ratio $a / b$ and the composant that contains the image $f(e)$ of the neutral element $e \in \mathcal{S}_{\mathcal{P}}$.

Our second theorem assures that the topological entropy is constant across any fixed homotopy class.

THEOREM 2. In the context of Theorem 1, the topological entropies of $f$ and $g$ coincide and are given by

$$
h(f)=h(g)=\log \max \{a, b\} .
$$

In particular, if $f$ is homotopic to a translation then the entropy is zero.

Finally, our third theorem shows that if $h(f)>0$, then $f$ is conjugate to $g$ after perhaps collapsing some $\operatorname{arcs}$ in $\mathcal{S}_{\mathcal{P}}$ to points.

TheOREm 3. In the context of Theorem 1 , if $a / b \neq 1$, then $f$ is semiconjugate to g; namely, there is a surjective continuous map $h: \mathcal{S}_{\mathcal{P}} \rightarrow \mathcal{S}_{\mathcal{P}}$ such that $h \circ f=g \circ h$. Moreover, $h^{-1}(z)$ is a point or an arc for any $z \in \mathcal{S}_{\mathcal{P}}$.

It is easy to see that the semi-conjugacy does not in general exist when $a / b=1$ (cf. [13]).

Let us mention that the above results show that the set of entropies exhibited by homeomorphisms of $\mathcal{S}_{\mathcal{P}}$ determines all the $\mathcal{P}$-recurrent primes and that this already determines $\mathcal{S}_{\mathcal{P}}$ up to a homeomorphism in the dynamically interesting case when $\mathcal{P}$ is a periodic sequence. Indeed, in [15, 1], it is shown that two solenoids $\mathcal{S}_{\mathcal{P}}$ and $\mathcal{S}_{\widetilde{\mathcal{P}}}$ are homeomorphic iff, after perhaps removing a finite number of terms, $\mathcal{P}$ and $\widetilde{\mathcal{P}}$ contain each prime the same number of times. In general, however, non-homeomorphic solenoids may exhibit the same entropies as exemplified by the pair $\mathcal{S}_{(2,3,5,7, \ldots)}$ and $\mathcal{S}_{(2,2,3,3,5,5,7,7, \ldots)}$-both solenoids admit only self-homeomorphisms of zero entropy.

The proofs of the three theorems hinge on constructing for an arbitrary homeomorphism of $\mathcal{S}_{\mathcal{P}}$ its lift to $\Lambda_{\mathcal{P}} \times \mathbb{R}$ where $\Lambda_{\mathcal{P}}$ is a cross section of $\mathcal{S}_{\mathcal{P}}$. The lift, although generally no longer a homeomorphism, is a skew product over the base $\Lambda_{\mathcal{P}}$ because it has to permute the composants $\{\omega\} \times \mathbb{R}$. Moreover, the base map on $\Lambda_{\mathcal{P}}$ is purely algebraic and universal across each homotopy class.

To indicate other ingredients of our arguments, we outline the contents of the sections to follow. In Section 2, we give a formal definition of $\mathcal{S}_{\mathcal{P}}$ and recall the standard identification of the cross section $\Lambda_{\mathcal{P}}$ of $\mathcal{S}_{\mathcal{P}}$ with an adding machine. Section 3 collects number-theoretical facts about $\Lambda_{\mathcal{P}}$ used in Section 4 to classify the algebraic homeomorphisms of $\mathcal{S}_{\mathcal{P}}$. Theorem 1 is shown in Section 5 by using "small cross sections" of $\mathcal{S}_{\mathcal{P}}$ to lift an arbitrary 
homeomorphism of $\mathcal{S}_{\mathcal{P}}$ to $\Lambda_{\mathcal{P}} \times \mathbb{R}$. A short Section 6 combines the results of Sections 4 and 5 to provide an explicit formula for such a lift. Section 7 establishes Theorem 2 by computing the entropy in $\Lambda_{\mathcal{P}} \times \mathbb{R}$ as the sum of the entropies in the fibers and in the base. This requires a suitable version of Bowen's formula from [4], which we prove in the appendix. Section 8 mimics Handel's [9] to implement Katok's idea of global shadowing in order to show Theorem 3. Finally, Section 9 develops the analogues of Theorems 1-3 for Knaster continua by lifting the homeomorphisms from $\mathcal{K}_{\mathcal{P}}$ to $\mathcal{S}_{\mathcal{P}}$.

To end, let us put our results into perspective $\left({ }^{2}\right)$. Solenoids are old and we inevitably included some known material hoping to make the presentation accessible even to a novice. The author's initial thrust came from conversations with Marcy Barge. In [3], a special case of Theorem 2 was established for $\mathcal{P}=\{n, n, \ldots\}$ and $f$ homotopic to a power of the very Frobenius homomorphism $g_{n}$. [3] also shows the formula for $h\left(g_{a / b}\right)$ for all $a, b$, which, however, goes back to [2] and belongs to a long line of works as cited in [14]. Theorem 1, in turn, is not original: it belongs to "folklore" among topologists and can be extracted from any of $[15,18,12,16,7]$; in particular, its version for maps fixing the unit element can be found on page 45 of [7]. A quick proof amounts to observing that a homeomorphism of a solenoid must permute the components according to its cohomological action on the dual group. Nevertheless, to present all three results from a unified point of view, we supply a careful elementary argument. Also, we should add that the analogue of Theorem 1 for $\mathcal{K}_{\mathcal{P}}$ with periodic $\mathcal{P}$ has been attributed in [3] to W. T. Watkins $\left({ }^{3}\right)$ and it follows from Lemma 9.5 of [6]. Theorem 3 seems genuinely new although it relies on tested tools of hyperbolic dynamics and is a natural extension of the classical result in $[8,9]$. We mention that our result complements [12], where the group of homeomorphisms of a solenoid was studied as a topological space. Finally, non-locally connected coverings like $\Lambda_{\mathcal{P}} \times \mathbb{R} \rightarrow \mathcal{S}_{\mathcal{P}}$ were used in [17]; however, our approach to homeomorphisms of $\mathcal{S}_{\mathcal{P}}$ via their (non-invertible) lifts to $\Lambda_{\mathcal{P}} \times \mathbb{R}$ seems original and is the key idea of this paper.

2. Preliminaries. We fix a sequence $\mathcal{P}=\left\{p_{0}, p_{1}, p_{2}, \ldots\right\}$ where $p_{k}>1$ for $k \geq 1$ are prime numbers, and $p_{0}=1$ is added for convenience. We define the $\mathcal{P}$-adic solenoid $\mathcal{S}_{\mathcal{P}}$ as the inverse limit space of the mappings $z \mapsto z^{p_{k}}$, $k=1,2, \ldots$, on the complex unit circle $\mathbb{S}:=\{z \in \mathbb{C}:|z|=1\}$; namely,

$$
\mathcal{S}_{\mathcal{P}}=\varliminf_{\lim }\left(z \mapsto z^{p_{k}}\right):=\left\{\left(z_{k}\right)_{k=0}^{\infty}: z_{k-1}=z_{k}^{p_{k}}, k \geq 1\right\} .
$$

$\left({ }^{2}\right)$ The author was greatly aided by feedback from the preliminary circulation of the manuscript and is especially grateful to M. Barge, L. Block, J. Keesling, P. Minc, V. Ssembatya, and the anonymous referee.

$\left({ }^{3}\right)$ Although we could not locate a written account. 
(This $\mathcal{S}_{\mathcal{P}}$ is homeomorphic to the geometric model discussed in the introduction, which justifies the abuse of notation.) Being a closed subgroup of the product $\prod_{k=0}^{\infty} \mathbb{S}, \mathcal{S}_{\mathcal{P}}$ is a compact abelian topological group (cf. $\S 10$ in Chapter II of [10]); we write $z \cdot \widetilde{z}$ for the product in $\mathcal{S}_{\mathcal{P}}$ and $e$ for the neutral element $(1,1,1, \ldots) . \mathcal{S}_{\mathcal{P}}$ is also a solenoidal group, meaning that there is a homomorphism $\Gamma: \mathbb{R} \rightarrow \mathcal{S}_{\mathcal{P}}$ with dense image, $\overline{\Gamma(\mathbb{R})}=\mathcal{S}_{\mathcal{P}}$. Explicitly,

$$
\Gamma(t):=\left(\exp \left(2 \pi i t /\left(p_{0} \ldots p_{k}\right)\right)\right)_{k=0}^{\infty}, \quad t \in \mathbb{R}
$$

$\Gamma$ generates a translation flow $T: \mathbb{R} \times \mathcal{S}_{\mathcal{P}} \rightarrow \mathcal{S}_{\mathcal{P}}, T^{t} z:=\Gamma(t) \cdot z$, which has an obvious cross section with return time 1 along a subgroup given by

$$
\Lambda_{\mathcal{P}}:=\left\{z=\left(z_{k}\right)_{k=0}^{\infty} \in \mathcal{S}_{\mathcal{P}}: z_{0}=1\right\}, \quad T^{1}\left(\Lambda_{\mathcal{P}}\right)=\Lambda_{\mathcal{P}}
$$

(i.e. there is $t \geq 0$ with $T^{t} z \in \Lambda_{\mathcal{P}}$ for any $z \in \mathcal{S}_{\mathcal{P}}$ and $\left\{t \in \mathbb{R}: T^{t} z \in \Lambda_{\mathcal{P}}\right\}$ $=\mathbb{Z}$ for $\left.z \in \Lambda_{\mathcal{P}}\right)$. The return map is the translation by $\gamma:=\Gamma(1)$,

$$
T:=\left.T^{1}\right|_{\Lambda_{\mathcal{P}}}: \Lambda_{\mathcal{P}} \rightarrow \Lambda_{\mathcal{P}}, \quad T \omega:=\gamma \cdot \omega
$$

and $\mathcal{S}_{\mathcal{P}}$ is homeomorphic to the suspension manifold of $T, \mathcal{S}_{\mathcal{P}} \cong \Lambda_{\mathcal{P}} \times[0,1] / \sim$ where $(\omega, 1) \sim(T \omega, 0)$ for $\omega \in \Lambda_{\mathcal{P}}$. Equivalently, $\mathcal{S}_{\mathcal{P}}$ is the orbit space of $\mathbb{Z}$ acting (discretely) on $\Lambda_{\mathcal{P}} \times \mathbb{R}$ so that $k \in \mathbb{Z}$ is assigned the map

$$
D^{k}:(\omega, x) \mapsto\left(T^{k} \omega, x-k\right) .
$$

We denote by $\pi$ the associated natural projection

$$
\pi: \Lambda_{\mathcal{P}} \times \mathbb{R} \rightarrow \Lambda_{\mathcal{P}} \times \mathbb{R} / \mathbb{Z} \cong \mathcal{S}_{\mathcal{P}},
$$

which is a covering and a homomorphism of topological groups $\left({ }^{4}\right)$ (cf. [17]). Being a covering, $\pi$ has the unique path lifting property and the homotopy lifting property (see Sec. 2, Chap. 2 in [19]); however, unlike in the usual setting, $\Lambda_{\mathcal{P}} \times \mathbb{R}$ is neither connected nor locally connected so that the standard lifting theorems do not apply. In particular, if $\kappa: \Lambda_{\mathcal{P}} \rightarrow \mathbb{Z}$ is any continuous function, then the map

$$
D^{\kappa(\cdot)}: \Lambda_{\mathcal{P}} \times \mathbb{R} \rightarrow \Lambda_{\mathcal{P}} \times \mathbb{R}, \quad D^{\kappa(\cdot)}:(\omega, x) \mapsto\left(T^{\kappa(\omega)} \omega, x-\kappa(\omega)\right),
$$

is clearly a covering transformation (i.e. $\pi \circ D^{\kappa(\cdot)}=\pi$ ), yet $D^{\kappa(\cdot)}$ is not invertible for non-constant $\kappa$. This (along with other features of $T$ ) is made transparent by viewing $T$ as an "adding machine", which we recall below.

Topologically, $\Lambda_{\mathcal{P}}$ is a $\mathcal{P}$-adic Cantor set: it is homeomorphic to $\mathcal{C}_{\mathcal{P}}:=$ $\prod_{k=1}^{\infty}\left\{0, \ldots, p_{k}-1\right\}$ via

$$
\mathcal{C}_{\mathcal{P}} \ni\left(d_{k}\right)_{k=1}^{\infty} \mapsto
$$

$$
\left(z_{k}\right)_{k=0}^{\infty}:=\exp \left(2 \pi i \frac{d_{1}+d_{2} p_{1}+d_{3} p_{1} p_{2}+\ldots+d_{k} p_{1} \ldots p_{k-1}}{p_{0} p_{1} \ldots p_{k}}\right) .
$$

$\left({ }^{4}\right) \mathcal{S}_{\mathcal{P}}=\Lambda_{\mathcal{P}} \times \mathbb{R} / I$ where $I$ is the subgroup $I=\left\{\left(\gamma^{k}, x-k\right): k \in \mathbb{Z}\right\}$. 
Here, we recognize the sequences $\left[d_{1} \ldots d_{k}\right]$ as the $\mathcal{P}$-adic representations of the integers

$$
s_{k}:=d_{1}+d_{2} p_{1}+d_{3} p_{1} p_{2}+\ldots+d_{k} p_{1} \ldots p_{k-1},
$$

and the group operation on $\Lambda_{\mathcal{P}}$ corresponds to the addition with carry performed on $\left[d_{1} \ldots d_{k}\right]$ 's. More formally, the correspondence $\left(z_{k}\right)_{k=0}^{\infty} \mapsto\left(s_{k}\right)_{k=1}^{\infty}$ is an isomorphism of $\Lambda_{\mathcal{P}}$ as a topological group with the inverse limit of (additive) cyclic groups,

$$
\Lambda_{\mathcal{P}} \cong \lim _{\longleftarrow} \mathbb{Z}_{p_{1}, \ldots, p_{k}},
$$

where the bonding maps $\mathbb{Z}_{p_{1} \ldots p_{k}} \rightarrow \mathbb{Z}_{p_{1} \ldots p_{k-1}}$ are given by

$$
s\left(\bmod p_{1} \ldots p_{k}\right) \mapsto s\left(\bmod p_{1} \ldots p_{k-1}\right), \quad k>1 .
$$

The action of $T$ corresponds to the addition with carry of $[1000 \ldots]$ and is given on $s \in \lim _{\longleftarrow} \mathbb{Z}_{p_{1}, \ldots, p_{k}}$ simply by

$$
T:\left(s_{k}\right) \mapsto\left(s_{k}+1\right) .
$$

It follows easily that the orbits of $T$ are dense in $\Lambda_{\mathcal{P}}$; in particular, $\Lambda_{\mathcal{P}}=$ $\overline{\left\{\gamma^{k}: k \in \mathbb{N}\right\}}$ and $\mathcal{S}_{\mathcal{P}}=\overline{\left\{T^{t} e: t \in \mathbb{R}\right\}}$.

To compute the entropy in Section 7, we shall use the metric $d_{\Lambda_{\mathcal{P}}}$ on $\Lambda_{\mathcal{P}}$ given in terms of the $\mathcal{P}$-adic representations by

$$
d_{\Lambda_{\mathcal{P}}}\left(\left[d_{k}\right]_{k=1}^{\infty},\left[\widetilde{d}_{k}\right]_{k=1}^{\infty}\right):=\exp \left(-\min \left\{k \geq 1: d_{k} \neq \widetilde{d}_{k} ; \infty\right\}\right) .
$$

Note that $d_{\Lambda_{\mathcal{P}}}$ is induced by a norm, $\left\|\left[d_{k}\right]\right\|_{\Lambda_{\mathcal{P}}}:=\exp \left(-\min \left\{k: d_{k} \neq 0\right\}\right)$, and therefore $d_{\Lambda_{\mathcal{P}}}$ is an invariant metric, i.e. the translations of $\Lambda_{\mathcal{P}}$ are isometries. On $\Lambda_{\mathcal{P}} \times \mathbb{R}$ and $\mathcal{S}_{\mathcal{P}}$, we put the corresponding invariant metrics:

$$
d_{\Lambda_{\mathcal{P}} \times \mathbb{R}}((\omega, x),(\widetilde{\omega}, \widetilde{x})):=\max \left\{d_{\Lambda_{\mathcal{P}}}(\omega, \widetilde{\omega}),|x-\widetilde{x}|\right\}
$$

and

$$
d_{\mathcal{S}_{\mathcal{P}}}(z, \widetilde{z}):=\min \left\{d_{\Lambda_{\mathcal{P}} \times \mathbb{R}}((\omega, x),(\widetilde{\omega}, \widetilde{x})): \pi(\omega, x)=z, \pi(\widetilde{\omega}, \widetilde{x})=\widetilde{z}\right\} .
$$

In this way, the deck map $D: \Lambda_{\mathcal{P}} \times \mathbb{R} \rightarrow \Lambda_{\mathcal{P}} \times \mathbb{R}$ becomes an isometry, and the covering projection $\pi: \Lambda_{\mathcal{P}} \times \mathbb{R} \rightarrow \mathcal{S}_{\mathcal{P}}$ becomes a local isometry.

3. Self-similarity of $\Lambda_{\mathcal{P}}$. Let a sequence $\mathcal{P}$ of primes be fixed as in the previous section. For determination of possible types of homeomorphisms of $\mathcal{S}_{\mathcal{P}}$ in Section 4, it matters how the number theory of $\mathcal{P}$ correlates with "selfsimilarity of $\Lambda_{\mathcal{P}}$ ". Precisely: for $a \in \mathbb{N}$, we have the associated Frobenius endomorphism

$$
\phi_{a}: \Lambda_{\mathcal{P}} \rightarrow \Lambda_{\mathcal{P}}, \quad \phi_{a}: \omega \mapsto \omega^{a} ;
$$

and we want to know that $\phi_{a}$ is a monomorphism onto a subgroup of index $a$ exactly when $a$ is $\mathcal{P}$-recurrent (i.e. any prime that divides $a$ appears in $\mathcal{P}$ infinitely many times). In fact, we shall need a bit more, which necessitates the following definitions. 
Let $\mathbb{P}$ be the set of all prime numbers, and let $\alpha_{\mathcal{P}}: \mathbb{P} \rightarrow\{0,1,2, \ldots, \infty\}$ be the function that counts primes in $\mathcal{P}$,

$$
\alpha_{\mathcal{P}}(p):=\#\left\{i \in \mathbb{N}: p_{i}=p\right\}, \quad p \in \mathbb{P} .
$$

For $a \in \mathbb{N}$, we have an analogous count of primes dividing $a, \alpha_{a}: \mathbb{P} \rightarrow$ $\{0,1, \ldots\}$,

$$
\alpha_{a}(p):=\max \left\{k \geq 0: p^{k} \text { divides } a\right\}, \quad p \in \mathbb{P} .
$$

The product $a=\prod_{p \in \mathbb{P}} p^{\alpha_{a}(p)}$ can be decomposed according to the number of times $p$ appears in $\mathcal{P}$ : the primes $p$ that repeat in $\mathcal{P}$ infinitely many times contribute

$$
a_{\mathcal{P}, \infty}:=\prod_{\alpha_{\mathcal{P}}(p)=\infty} p^{\alpha_{a}(p)}
$$

the primes $p$ that repeat in $\mathcal{P}$ finitely many times contribute

$$
a_{\mathcal{P},+}:=\prod_{0 \leq \alpha \mathcal{P}(p)<\infty} p^{\min \left\{\alpha_{a}(p), \alpha_{\mathcal{P}}(p)\right\}}
$$

and there are primes $p$ that do not occur in $\mathcal{P}$ or occur in $\mathcal{P}$ fewer times than in $a$,

$$
a_{\mathcal{P},-}:=\prod_{0 \leq \alpha_{\mathcal{P}}(p)<\infty} p^{\alpha_{a}(p)-\min \left\{\alpha_{a}(p), \alpha_{\mathcal{P}}(p)\right\}} .
$$

The number

$$
a_{\mathcal{P}}:=a_{\mathcal{P}, \infty} a_{\mathcal{P},+}
$$

is the greatest common divisor of $a$ and the (infinite) product $\prod_{p_{i} \in \mathcal{P}} p_{i}$. We have

$$
a=a_{\mathcal{P}} a_{\mathcal{P},-}=a_{\mathcal{P}, \infty} a_{\mathcal{P},+} a_{\mathcal{P},-}
$$

and $a \in \mathbb{N}$ is $\mathcal{P}$-recurrent iff $a=a_{\mathcal{P}, \infty}$.

FACT 3.1. For $a \in \mathbb{N}$, the kernel of $\phi_{a}$ has cardinality $\left|\operatorname{ker}\left(\phi_{a}\right)\right|=a_{\mathcal{P},+}$. In particular, $\phi_{a}$ is a monomorphism iff $a=a_{\mathcal{P}, \infty} a_{\mathcal{P},-}$, i.e. every prime in $\mathcal{P}$ that divides a repeats in $\mathcal{P}$ infinitely many times.

We shall argue for the representation of $\phi_{a}$ on $\lim _{\longleftarrow} \mathbb{Z}_{p_{1}, \ldots, p_{k}} \equiv \Lambda_{\mathcal{P}}$ (see Section 2) given by

$$
\psi_{a}: \lim _{\longleftarrow} \mathbb{Z}_{p_{1}, \ldots, p_{k}} \rightarrow \varliminf_{\lim _{1}} \mathbb{Z}_{p_{1}, \ldots, p_{k}}, \quad \psi_{a}: s=\left(s_{k}\right)_{k=1}^{\infty} \mapsto a s:=\left(a s_{k}\right)_{k=1}^{\infty} .
$$

Proof of Fact 3.1. Let $k_{0}$ be large enough so that $a_{\mathcal{P}}$ divides $p_{1} \ldots p_{k_{0}}$ and no prime $p_{k}$ divides $a_{\mathcal{P},+}$ for $k>k_{0}$. In particular, $a_{\mathcal{P},-}$ and $p_{1} \ldots p_{k} / a_{\mathcal{P}}$ are co-prime for $k>k_{0}$. Consider $s \in \operatorname{ker}\left(\psi_{a}\right)$. We have

$$
a s_{k}=a_{\mathcal{P},-} a_{\mathcal{P}} s_{k} \equiv 0\left(\bmod p_{1} \ldots p_{k}\right), \quad k \geq 1 .
$$

Thus, for $k>k_{0}, a_{\mathcal{P},-} s_{k} \equiv 0\left(\bmod p_{1} \ldots p_{k} / a_{\mathcal{P}}\right)$ and, by the choice of $k_{0}$,

$$
s_{k} \equiv 0\left(\bmod p_{1} \ldots p_{k} / a_{\mathcal{P}}\right) \text {. }
$$


Also, for a fixed $k>k_{0}$ and $l>k$ large enough, $a_{\mathcal{P}, \infty}$ divides $p_{k+1} \ldots p_{l}$ so that $p_{1} \ldots p_{k} / a_{\mathcal{P},+}$ divides $p_{1} \ldots p_{l} / a_{\mathcal{P}}$. The congruence (3.1) (applied for $k=l)$ yields that $s_{l} \equiv 0\left(\bmod p_{1} \ldots p_{k} / a_{\mathcal{P},+}\right)$ and thus $s_{k} \equiv s_{l} \equiv 0$ $\left(\bmod p_{1} \ldots p_{k} / a_{\mathcal{P},+}\right)$ since $s \in \lim \mathbb{Z}_{p_{1}, \ldots, p_{k}}$. This shows that an arbitrary $s \in \operatorname{ker}\left(\psi_{a}\right)$ is of the form

$$
s_{k}=x_{k} p_{1} \ldots p_{k} / a_{\mathcal{P},+} \in \mathbb{Z}_{p_{1} \ldots p_{k}}, \quad k>k_{0},
$$

where $x_{k} \in \mathbb{Z}_{a_{\mathcal{P},+}}$. As a result there are at most $a_{\mathcal{P},+}=\left|\mathbb{Z}_{a_{\mathcal{P},+}}\right|$ elements in $\operatorname{ker}\left(\psi_{a}\right)$ (because they would all have to differ on some fixed coordinate $s_{k}$ for sufficiently large $k$ ).

To see that $\left|\operatorname{ker}\left(\psi_{a}\right)\right|=a_{\mathcal{P},+}$, for any $x_{k_{0}} \in \mathbb{Z}_{a_{\mathcal{P},+}}$ we exhibit $s \in \operatorname{ker}\left(\psi_{a}\right)$ with $s_{k_{0}}=x_{k_{0}} p_{1} \ldots p_{k_{0}} / a_{\mathcal{P},+}$. For $k<k_{0}$, set $s_{k}:=s_{k_{0}}\left(\bmod p_{1} \ldots p_{k}\right)$. For $k \geq k_{0}$, since $p_{k+1}$ is co-prime $a_{\mathcal{P},+}$ by the choice of $k_{0}$, we can solve recursively for $x_{k}$ 's the equations

$$
x_{k+1} p_{k+1} \equiv x_{k}\left(\bmod a_{\mathcal{P},+}\right), \quad k \geq k_{0} ;
$$

and we can set $s_{k}:=x_{k} p_{1} \ldots p_{k} / a_{\mathcal{P},+}$. The recurrence (3.2) guarantees that $s_{k+1} \equiv s_{k}\left(\bmod p_{1} \ldots p_{k}\right)$ for all $k \geq 1$, i.e. that $s \in \varliminf_{\longleftarrow} \mathbb{Z}_{p_{1}, \ldots, p_{k}}$. It is also immediate that $\psi_{a}(s)=0$.

FACT 3.2. For $a \in \mathbb{N}$, the image $\phi_{a}\left(\Lambda_{\mathcal{P}}\right)=\Lambda_{\mathcal{P}}^{a} \subset \Lambda_{\mathcal{P}}$ is a subgroup of index

$$
\left[\Lambda_{\mathcal{P}}: \Lambda_{\mathcal{P}}^{a}\right]=a_{\mathcal{P}}=a_{\mathcal{P}, \infty} a_{\mathcal{P},+} .
$$

In particular, $\phi_{a}$ is an epimorphism iff $a=a_{\mathcal{P},-}$, i.e. no prime in $\mathcal{P} d i$ vides a.

Proof. As before, take $k_{0}$ large enough so that $a_{\mathcal{P}}$ divides $p_{1} \ldots p_{k_{0}}$. Note that $a_{\mathcal{P},-}$ and $p_{1} \ldots p_{k} / a_{\mathcal{P}}$ are co-prime for $k>k_{0}$. Consider a homomorphism $h: \lim _{p_{1}, \ldots, p_{k}} \rightarrow \mathbb{Z}_{a_{\mathcal{P}}}$ given by $h: s \mapsto s_{k_{0}}\left(\bmod a_{\mathcal{P}}\right)$. This is an epimorphism because, given $x \in \mathbb{Z}_{a_{\mathcal{P}}}$, we clearly have $h(s)=x$ for $s=\left(s_{k}\right)_{k=1}^{\infty}:=\left(x\left(\bmod p_{1} \ldots p_{k}\right)\right)_{k=1}^{\infty}$. It follows that $\left[\lim _{\mathbb{Z}_{1}, \ldots, p_{k}}\right.$ : $\operatorname{ker}(h)]=a_{\mathcal{P}}$. To finish the proof, we show that $\operatorname{ker}(h)=\psi_{a}\left(\varliminf_{\longleftarrow} \mathbb{Z}_{p_{1}, \ldots, p_{k}}\right)$.

If $s \in \psi_{a}\left(\varliminf_{\mathbb{Z}_{1}, \ldots, p_{k}}\right)$, then $s \in \operatorname{ker}(h)$ because $s_{k_{0}} \equiv 0(\bmod a)$. For the other inclusion, observe that if $h(s)=0$, then $s_{k_{0}} \equiv 0\left(\bmod a_{\mathcal{P}}\right)$; and $s_{k} \equiv 0\left(\bmod a_{\mathcal{P}}\right)$ for $k \geq k_{0}$ because $s_{k} \equiv s_{k_{0}}\left(\bmod p_{1} \ldots p_{k_{0}}\right)$ and $a_{\mathcal{P}}$ divides $p_{1} \ldots p_{k_{0}}$. Hence, $s_{k}=x_{k} a_{\mathcal{P}}\left(\bmod p_{1} \ldots p_{k}\right)$ for some $x_{k} \in \mathbb{Z}_{p_{1} \ldots p_{k} / a_{\mathcal{P}}}, k \geq$ $k_{0}$. Because $a_{\mathcal{P},-}$ and $p_{1} \ldots p_{k} / a_{\mathcal{P}}$ are co-prime for $k>k_{0}$, we can solve the equations $a_{\mathcal{P},-} y_{k} \equiv x_{k}\left(\bmod p_{1} \ldots p_{k} / a_{\mathcal{P}}\right)$ for $y_{k} \in \mathbb{Z}_{p_{1} \ldots p_{k} / a_{\mathcal{P}}}, k \geq k_{0}$. It follows that $a_{\mathcal{P},-} a_{\mathcal{P}} y_{k} \equiv a_{\mathcal{P}} x_{k} \equiv s_{k}\left(\bmod p_{1} \ldots p_{k}\right)$ so that $\psi_{a}(y)=s$, i.e. $s \in \psi_{a}\left(\lim _{\longleftarrow} \mathbb{Z}_{p_{1}, \ldots, p_{k}}\right)$.

4. Algebraic homeomorphisms. In this section, we list the self-homeomorphisms of solenoids that are to serve as the representatives of the 
homotopy classes. (We shall proceed in a completely elementary fashion that avoids Pontryagin's duality.)

The inverse. Taking inverse with respect to the group operation in $\mathcal{S}_{\mathcal{P}}$ yields a homeomorphism

$$
r: \mathcal{S}_{\mathcal{P}} \rightarrow \mathcal{S}_{\mathcal{P}}, \quad r: z \mapsto z^{-1} .
$$

The corresponding map on the cover,

$$
R: \Lambda_{\mathcal{P}} \times \mathbb{R} \rightarrow \Lambda_{\mathcal{P}} \times \mathbb{R}, \quad R:(\omega, x) \mapsto\left(\omega^{-1},-x\right),
$$

is a lift of $r$.

Translations. For $w \in \mathcal{S}_{\mathcal{P}}$, the translation by $w$ yields a homeomorphism

$$
s_{w}: \mathcal{S}_{\mathcal{P}} \rightarrow \mathcal{S}_{\mathcal{P}}, \quad z \mapsto w \cdot z .
$$

If $(\sigma, t) \in \Lambda_{\mathcal{P}} \times \mathbb{R}$ is a lift of $w, \pi(\sigma, t)=w$, then

$$
S_{(\sigma, t)}: \Lambda_{\mathcal{P}} \times \mathbb{R} \rightarrow \Lambda_{\mathcal{P}} \times \mathbb{R}, \quad S_{(\sigma, t)}:(\omega, x) \mapsto(\omega \cdot \sigma, x+t),
$$

is a lift of $s_{w}$.

Frobenius automorphisms. For $a \in \mathbb{N}$, we have the Frobenius endomorphism

$$
g_{a}: \mathcal{S}_{\mathcal{P}} \rightarrow \mathcal{S}_{\mathcal{P}}, \quad g_{a}: z \mapsto z^{a},
$$

which lifts to the corresponding Frobenius endomorphism on $\Lambda_{\mathcal{P}} \times \mathbb{R}$,

$$
G_{a}: \Lambda_{\mathcal{P}} \times \mathbb{R} \rightarrow \Lambda_{\mathcal{P}} \times \mathbb{R}, \quad G_{a}:(\omega, x) \mapsto\left(\phi_{a}(\omega), a x\right) .
$$

$\left(\phi_{a}: \Lambda_{\mathcal{P}} \rightarrow \Lambda_{\mathcal{P}}, \phi_{a}(\omega)=\omega^{a}\right.$ was discussed in Section 3.)

Lemma 4.1. The Frobenius endomorphism $g_{a}$ is a homeomorphism of $\mathcal{S}_{\mathcal{P}}$ iff $a$ is $\mathcal{P}$-recurrent, i.e. $a=a_{\mathcal{P}, \infty}$.

The idea is that $g_{a}$ is a homeomorphism exactly when $\phi_{a}$ is $1-1$ and $\left[\Lambda_{\mathcal{P}}: \phi_{a}\left(\Lambda_{\mathcal{P}}\right)\right]=a$, which will translate to $a=a_{\mathcal{P}, \infty}$ by the results of the previous section. The first condition is clear because $\phi_{a}=\left.g_{a}\right|_{\Lambda_{\mathcal{P}}}$, and the second condition is to prevent the fundamental domain $\Lambda_{\mathcal{P}} \times[0,1]$ from overlapping in the image of $G_{a}$.

Proof of Lemma 4.1. Observe that $g_{a}$ is surjective for any $a \in \mathbb{N}$ because $g_{a}(\Gamma(t))=\Gamma(a t)$ for $t \in \mathbb{R}$ and $\overline{\Gamma(a \mathbb{R})}=\mathcal{S}_{\mathcal{P}}$.

Suppose that $a=a_{\mathcal{P}, \infty}$. We first prove that $g_{a}$ is a homeomorphism by deriving an explicit formula for its (lifted) inverse. (This may not be the shortest route but the formula will be crucial in all subsequent sections.) From Fact $3.2, \Lambda_{\mathcal{P}}$ decomposes into a disjoint union of $a$ clopen cosets

$$
\Lambda_{\mathcal{P}}=\Lambda_{\mathcal{P}}^{a} \cup \gamma \cdot \Lambda_{\mathcal{P}}^{a} \cup \ldots \cup \gamma^{a-1} \cdot \Lambda_{\mathcal{P}}^{a} .
$$


Let $I_{a}: \Lambda_{\mathcal{P}} \rightarrow \mathbb{Z}_{a} \simeq \Lambda_{\mathcal{P}} / \Lambda_{\mathcal{P}}^{a}$ be the natural projection so that $\omega \in \gamma^{I_{a}(\omega)} \Lambda_{\mathcal{P}}^{a}$ for $\omega \in \Lambda_{\mathcal{P}}$. Define $G_{1 / a}: \Lambda_{\mathcal{P}} \times \mathbb{R} \rightarrow \Lambda_{\mathcal{P}} \times \mathbb{R}$ by the formula

$$
G_{1 / a}:(\omega, x) \mapsto\left(\phi_{a}^{-1}\left(\gamma^{-I_{a}(\omega)} \cdot \omega\right), \frac{x+I_{a}(\omega)}{a}\right) .
$$

$G_{1 / a}$ is manifestly continuous; and we claim that $G_{1 / a}$ descends to a continuous map $g_{1 / a}: \mathcal{S}_{\mathcal{P}} \rightarrow \mathcal{S}_{\mathcal{P}}$. It suffices to see that, given $(\omega, x) \in$ $\Lambda_{\mathcal{P}} \times \mathbb{R}, G_{1 / a} \circ D(\omega, x)=D^{k} \circ G_{1 / a}(\omega, x)$ for some $k \in \mathbb{N}$ :

If $I_{a}(\omega)<a-1$, so that $I_{a}(\gamma \cdot \omega)=I_{a}(\omega)+1$, then

$$
\begin{aligned}
G_{1 / a} \circ D(\omega, x) & =G_{1 / a}(\gamma \cdot \omega, x-1) \\
& =\left(\phi_{a}^{-1}\left(\gamma^{-I_{a}(\gamma \cdot \omega)} \cdot \gamma \cdot \omega\right), \frac{x-1+I_{a}(\gamma \cdot \omega)}{a}\right) \\
& =\left(\phi_{a}^{-1}\left(\gamma^{-I_{a}(\omega)} \cdot \omega\right), \frac{x+I_{a}(\omega)}{a}\right)=G_{1 / a}(\omega, x) .
\end{aligned}
$$

If $I_{a}(\omega)=a-1$, so that $\gamma \cdot \omega \in \Lambda_{\mathcal{P}}$, then

$$
G_{1 / a} \circ D(\omega, x)=G_{1 / a}(\gamma \cdot \omega, x-1)=\left(\phi_{a}^{-1}(\gamma \cdot \omega), \frac{x-1}{a}\right)
$$

coincides with

$$
\begin{aligned}
D \circ G_{1 / a}(\omega, x) & =\left(\gamma \cdot \phi_{a}^{-1}\left(\gamma^{-a+1} \cdot \omega\right), \frac{x+a-1}{a}-1\right) \\
& =\left(\gamma \cdot \phi_{a}^{-1}\left(\gamma^{-a+1} \cdot \omega\right), \frac{x-1}{a}\right)
\end{aligned}
$$

because $\gamma \cdot \phi_{a}^{-1}\left(\gamma^{-a+1} \cdot \omega\right)=\phi_{a}^{-1}(\gamma \cdot \omega)$, which is easily verified by applying $\phi_{a}$ to both sides and simplifying. Thus, indeed, $g_{1 / a}$ is well defined.

Note that $G_{1 / a}$ is a left inverse of $G_{a}, G_{1 / a} \circ G_{a}=\mathbf{I d}_{\Lambda_{\mathcal{P}} \times \mathbb{R}}$. Therefore, since $g_{a}$ is surjective (unlike $G_{a}$ ), $g_{1 / a}$ is the (two-sided) inverse of $g_{a}$, which makes $g_{a}$ a homeomorphism.

To prove the other implication, suppose that $g_{a}$ is a homeomorphism. If $a=q_{1} \ldots q_{m}$ is the prime decomposition of $a$, then $g_{a}=g_{q_{1}} \circ \ldots \circ g_{q_{m}}$ where the $g_{q_{i}}$ 's are homeomorphisms as well. Consider a single homeomorphism $g_{q}$ for a prime $q=q_{i}$. If $q$ appears in $\mathcal{P}$, then $q$ must be $\mathcal{P}$-recurrent since otherwise $\phi_{q}=\left.g_{q}\right|_{\Lambda_{\mathcal{P}}}$ is not 1-1 by Fact 3.1. If $q$ does not appear in $\mathcal{P}$, then Facts 3.2 and 3.1 imply that $\phi_{q}: \Lambda_{\mathcal{P}} \rightarrow \Lambda_{\mathcal{P}}$ is a homeomorphism and so are the restrictions

$$
\left.g_{q}\right|_{\pi\left(\Lambda_{\mathcal{P}} \times\{k / q\}\right)}: \pi\left(\Lambda_{\mathcal{P}} \times\{k / q\}\right) \rightarrow \Lambda_{\mathcal{P}}, \quad k=0, \ldots, q-1 .
$$

Therefore, each point of $\Lambda_{\mathcal{P}}$ has (exactly) $q$ preimages, which contradicts $g_{q}$ being a homeomorphism. This shows that any prime factor $q_{i}$, and thus $a$, is $\mathcal{P}$-recurrent. 
By combining the Frobenius automorphisms, their inverses, translations, and possibly the inverse involution we arrive at the affine homeomorphisms of $\mathcal{S}_{\mathcal{P}}$-among which those that map $\Lambda_{\mathcal{P}}$ to itself we shall call normalized affine homeomorphisms.

Definition 4.1. Suppose that $\sigma \in \Lambda_{\mathcal{P}}$ and $a, b \in \mathbb{N}$ are co-prime and $\mathcal{P}$-recurrent. We define a homeomorphism

$$
g_{a / b, \sigma}: \mathcal{S}_{\mathcal{P}} \rightarrow \mathcal{S}_{\mathcal{P}}
$$

to be the factor of $G_{a / b, \sigma}: \Lambda_{\mathcal{P}} \times \mathbb{R} \rightarrow \Lambda_{\mathcal{P}} \times \mathbb{R}$ given by

$$
G_{a / b, \sigma}:(\omega, x) \mapsto\left(\sigma \cdot \phi_{a / b}(\omega), \frac{a\left(x+I_{b}(\omega)\right)}{b}\right)
$$

where

$$
\phi_{a / b}(\omega):=\phi_{a} \circ \phi_{b}^{-1}\left(\gamma^{-I_{b}(\omega)} \cdot \omega\right)
$$

and $I_{b}: \Lambda_{\mathcal{P}} \rightarrow \mathbb{Z}_{b}$ is determined by $\gamma^{-I_{b}(\omega)} \cdot \omega \in \Lambda_{\mathcal{P}}^{b}$.

A map $g: \mathcal{S}_{\mathcal{P}} \rightarrow \mathcal{S}_{\mathcal{P}}$ is called a normalized affine homeomorphism if $g=g_{a / b, \sigma}$ or $g=r \circ g_{a / b, \sigma}$ for some $a, b, \sigma$ as above.

A map $g: \mathcal{S}_{\mathcal{P}} \rightarrow \mathcal{S}_{\mathcal{P}}$ is called an affine homeomorphism if $g=T^{t} \circ g_{a / b, \sigma}$ or $g=r \circ T^{t} \circ g_{a / b, \sigma}$ for some $a, b, \sigma$ as above and $t \in \mathbb{R}$.

Note that $g_{a}$ and $g_{b}$ commute so that $g_{a / b, \sigma}=s_{\sigma} \circ g_{a} \circ g_{b}^{-1}=s_{\sigma} \circ g_{b}^{-1} \circ g_{a}$. Also, if $\widetilde{a}$ and $\widetilde{b}$ fail to be co-prime, one may define $g_{\widetilde{a} / \widetilde{b}, \sigma}:=g_{a / b, \sigma}$ where $a / b=\widetilde{a} / \widetilde{b}$ is the reduced fraction-which allows one to write $\left({ }^{5}\right)$

$$
t g_{a_{1} / b_{1}, \sigma_{1}} \circ g_{a_{2} / b_{2}, \sigma_{2}}=g_{a_{1} / b_{1} \cdot a_{2} / b_{2}, \sigma_{1} \cdot \phi_{a_{1} / b_{1}}\left(\sigma_{2}\right)}, \quad g_{a / b, \sigma}^{-1}=g_{b / a, \phi_{b / a}(\sigma)} .
$$

At the same time, keep in mind that if $b \neq 1$, then $G_{a / b, \sigma}=S_{\sigma} \circ G_{a} \circ G_{1 / b} \neq$ $S_{\sigma} \circ G_{1 / b} \circ G_{a}$, and $G_{a / b, \sigma}$ is neither 1-1 nor surjective.

REMARK 4.1. The formula (4.4) takes on a more friendly appearance when interpreted on $\lim _{\mathbb{Z}_{p_{1}, \ldots, p_{k}}} \cong \Lambda_{\mathcal{P}}$. Namely, if $w \in \varliminf_{\mathcal{l}} \mathbb{Z}_{p_{1}, \ldots, p_{k}}$ corresponds to $\sigma \in \Lambda_{\mathcal{P}}, a, b \in \mathbb{N}$ are co-prime and $b$ is $\mathcal{P}$-recurrent, then $\sigma \cdot \phi_{a / b}: \Lambda_{\mathcal{P}} \rightarrow \Lambda_{\mathcal{P}}$ is conjugate to $w+\psi_{a / b}: \varliminf_{\longleftarrow} \mathbb{Z}_{p_{1}, \ldots, p_{k}} \rightarrow \varliminf_{\longleftarrow} \mathbb{Z}_{p_{1}, \ldots, p_{k}}$ given by

$$
\left(w+\psi_{a / b}\right)(s)_{k}=w_{k}+a\left(s_{k} \div b\right)\left(\bmod p_{1} \ldots p_{k}\right), \quad s \in \lim _{\longleftarrow} \mathbb{Z}_{p_{1}, \ldots, p_{k}},
$$

valid for $k$ large enough to guarantee that $b$ divides $p_{1} \ldots p_{k}$. (Here the symbol $\div$ stands for integer division.)

We finish by classifying the affine homeomorphisms with respect to homotopy.

$\left({ }^{5}\right)$ That is, the affine homeomorphisms are a skew product of the group of $\mathcal{P}$-adic fractions and $\mathcal{S}_{\mathcal{P}}$. 
LEMmA 4.2. Two normalized affine homeomorphisms $g_{a / b, \sigma}$ and $g_{a_{1} / b_{1}, \sigma_{1}}$ are homotopic iff $a=a_{1}, b=b_{1}$, and $T^{n} \sigma=\sigma_{1}$ for some $n \in \mathbb{Z}$.

Proof. To prove necessity, it suffices to argue for $a_{1}=b_{1}=1$ and $\sigma_{1}=e$ (by (4.5)). Suppose that $f_{t}: \mathcal{S}_{\mathcal{P}} \rightarrow \mathcal{S}_{\mathcal{P}}, t \in[0,1]$, is a homotopy such that $f_{0}=g_{a / b, \sigma}$ and $f_{1}=$ Id. Being a covering, $\pi$ has the homotopy lifting property so that there is a homotopy $F_{t}: \Lambda_{\mathcal{P}} \times \mathbb{R} \rightarrow \Lambda_{\mathcal{P}} \times \mathbb{R}, t \in[0,1]$, covering $f_{t}$ and such that $F_{0}=G_{a / b, \sigma}$. Being a lift of $f_{1}=\mathbf{I d}, F_{1}$ must be a covering transformation, i.e. $F_{1}=D^{\kappa(\cdot)}$ for some continuous function $\kappa: \Lambda_{\mathcal{P}} \rightarrow \mathbb{Z}$ (cf. Section 2). Now, if $\mathrm{pr}_{\mathbb{R}}: \Lambda_{\mathcal{P}} \times \mathbb{R} \rightarrow \mathbb{R}$ is the projection on the second factor, then the compactness of $\Lambda_{\mathcal{P}}$ implies that $f_{t}$ has a bounded displacement; namely,

$$
\operatorname{pr}_{\mathbb{R}}\left(F_{0}(\omega, x)\right)-\operatorname{pr}_{\mathbb{R}}\left(F_{1}(\omega, x)\right)|=| a\left(x+I_{b}(\omega)\right) / b-(x-\kappa(\omega)) \mid<C
$$

for some $C>0$ independent of $x \in \mathbb{R}$ and $\omega \in \Lambda_{\mathcal{P}}$. It follows (by considering $x \rightarrow \infty)$ that $a / b=1$, i.e. $a=1$ and $b=1$. Moreover $-\Lambda_{\mathcal{P}}$ being totally disconnected $-F_{t}$ must permute the leaves $\omega \times \mathbb{R}$ in $\Lambda_{\mathcal{P}} \times \mathbb{R}$, which is to say that the projection $\operatorname{pr}_{\Lambda_{\mathcal{P}}}\left(F_{t}(\omega, x)\right)$ onto $\Lambda_{\mathcal{P}}$ is constant over the range $(t, x) \in[0,1] \times \mathbb{R}$, i.e. $\sigma \cdot \omega=\gamma^{\kappa(\omega)} \cdot \omega$ for all $\omega \in \Lambda_{\mathcal{P}}$. In this way, $\kappa(\cdot)$ must be constant and $\sigma=\gamma^{\kappa}$, which finishes the proof of necessity.

For sufficiency, observe that the flow $T^{t}$ provides isotopies from $g_{a / b, \sigma}$ to $g_{a / b, \gamma^{k} \cdot \sigma}$ and backwards.

5. Homotopy classes (proof of Theorem 1). In this section, we fix a homeomorphism $f: \mathcal{S}_{\mathcal{P}} \rightarrow \mathcal{S}_{\mathcal{P}}$ and construct an isotopy of $f$ to an affine homeomorphism $g$ of $\mathcal{S}_{\mathcal{P}}$ in order to prove Theorem 1 .

We first record the simple fact that $f$ is an orbit equivalence between the flow $T^{t}$ and itself or $T^{t}$ and its inverse, $T^{-t}$. (This suggests that the homotopy type of $f$ is solely determined by the way $f$ permutes the orbits of $T^{t}$.)

FACT 5.3. For every $z \in \mathcal{S}_{\mathcal{P}}$, there is a homeomorphism $\tau: \mathbb{R} \rightarrow \mathbb{R}$ such that

$$
f \circ T^{t} z=T^{\tau(t)} \circ f(z), \quad t \in \mathbb{R} .
$$

Depending on whether $\tau(t)$ is increasing or decreasing we shall call $f$ orientation preserving or orientation reversing (which $z$ is used is irrelevant because $\left\{T^{t}(z): t \in \mathbb{R}\right\}$ winds densely in $\left.\mathcal{S}_{\mathcal{P}}\right)$. At the expense of replacing $f$ with $r \circ f$, we may assume that $f$ is orientation preserving.

Proof of Fact 5.3. Fix $z \in \mathcal{S}_{\mathcal{P}}$. By path lifting, we have $\alpha: \mathbb{R} \rightarrow \Lambda_{\mathcal{P}} \times \mathbb{R}$ such that $\pi \circ \alpha(t)=f \circ T^{t} z$ for $t \in \mathbb{R}$. Because $\Lambda_{\mathcal{P}}$ is totally disconnected, $\operatorname{pr}_{\Lambda_{\mathcal{P}}} \circ \alpha$ is constant and so $\alpha(t)=(\omega, \tau(t))$ for some $\tau: \mathbb{R} \rightarrow \mathbb{R}$ and some $\omega \in \Lambda_{\mathcal{P}}$. Now, $\tau$ is $1-1$ because $f$ is $1-1$. Also, $\tau(\mathbb{R})=\mathbb{R}$ because otherwise $\pi(\{(\omega, \tau(t)): \pm t>0\})=\left\{f \circ T^{t} z: \pm t>0\right\}$ would not be dense in $\mathcal{S}_{\mathcal{P}}$. 
In order to lift $f$ to a map of $\Lambda_{\mathcal{P}} \times \mathbb{R}$, we shall use "small cross sections of $T^{t}$ ". For $N \in \mathbb{N}$, let $c:=p_{1} \ldots p_{N}$. Clearly, $c=c_{\mathcal{P}}$ so that $\left[\Lambda_{\mathcal{P}}: \Lambda_{\mathcal{P}}^{c}\right]=c$ by Fact 3.2. Hence, $\Lambda_{\mathcal{P}}^{c}$ is a cross section of the flow $T^{t}$ with constant return time $c$. Moreover, since $\Lambda_{\mathcal{P}}^{c}=\left\{\omega \in \Lambda_{\mathcal{P}}: \omega_{i}=1, \forall 0 \leq i \leq N\right\}$, we see that $\lim _{N \rightarrow \infty} \operatorname{diam}\left(\Lambda_{\mathcal{P}}^{c}\right)=0$ (with respect to any compatible metric). In this way, we can find $N \in \mathbb{N}, \varepsilon, \delta \in(0,1 / 4)$, and $x_{0} \in(0,1)$ such that

$$
\begin{gathered}
f \circ \pi\left(\Lambda_{\mathcal{P}}^{c} \times(-\delta, \delta)\right) \subset \pi\left(\Lambda_{\mathcal{P}} \times\left(x_{0}-\varepsilon, x_{0}+\varepsilon\right)\right), \\
\operatorname{diam}\left(\operatorname{pr}_{0} \circ f^{-1} \circ \pi\left(\omega \times\left(x_{0}-\varepsilon, x_{0}+\varepsilon\right)\right)\right)<1 / 2, \quad \omega \in \Lambda_{\mathcal{P}}, \\
\operatorname{dist}\left(\operatorname{pr}_{0} \circ f\left(z_{1}\right), \operatorname{pr}_{0} \circ f\left(z_{2}\right)\right)<1 / 4 \quad \text { for } z_{1} \cdot z_{2}^{-1} \in \Lambda_{\mathcal{P}}^{c}, z_{1}, z_{2} \in \mathcal{S}_{\mathcal{P}},
\end{gathered}
$$

where $\operatorname{pr}_{0}: \mathcal{S}_{\mathcal{P}} \rightarrow \mathbb{S}$ is the projection on the zeroth coordinate, $\operatorname{pr}_{0}\left(\left(z_{i}\right)_{i=0}^{\infty}\right)$ $=z_{0}$, and the metric on $\mathbb{S}$ is induced from $\mathbb{R}$ via the exponential $t \mapsto$ $\exp (2 \pi i t)$.

In particular, $\Lambda_{\mathcal{P}}^{c}$ is chosen to be a small enough cross section so that a localized isotopy will easily "straighten" $f\left(\Lambda_{\mathcal{P}}^{c}\right)$ and put it into a fiber of $\operatorname{pr}_{0}: \mathcal{S}_{\mathcal{P}} \rightarrow \mathbb{S}$, which can then be further isotoped into $\Lambda_{\mathcal{P}}$ via $T^{t}$. This is formalized in the first step below.

STEP 1: Construct an isotopy of $f$ to $\bar{f}$ such that $\bar{f}\left(\Lambda_{\mathcal{P}}^{c}\right) \subset \Lambda_{\mathcal{P}}$.

Construction. From (5.1), $f\left(\Lambda_{\mathcal{P}}^{c}\right)$ is evenly covered by $\pi$; in particular, we have a well defined and continuous $\xi:=\left.\operatorname{pr}_{\Lambda_{\mathcal{P}}} \circ \widetilde{\pi}^{-1} \circ f\right|_{\Lambda_{\mathcal{P}}^{c}}: \Lambda_{\mathcal{P}}^{c} \rightarrow \Lambda_{\mathcal{P}}$ and $\theta=\left.\operatorname{pr}_{\mathbb{R}} \circ \widetilde{\pi}^{-1} \circ f\right|_{\Lambda_{\mathcal{P}}^{c}}: \Lambda_{\mathcal{P}}^{c} \rightarrow \mathbb{R}$ where $\widetilde{\pi}:=\left.\pi\right|_{\Lambda_{\mathcal{P}} \times\left(x_{0}-\varepsilon, x_{0}+\varepsilon\right)}$. Clearly, $\left.f\right|_{\Lambda_{\mathcal{P}}^{c}}(\omega)=\pi(\xi(\omega), \theta(\omega))$. Observe that $\xi$ is 1-1. Indeed, if $\omega=\xi\left(\omega_{1}\right)=\xi\left(\omega_{2}\right)$ for $\omega_{1}, \omega_{2} \in \Lambda_{\mathcal{P}}^{c}$, then (5.1) implies that both $\omega_{1}$ and $\omega_{2}$ are contained in $f^{-1} \circ \pi\left(\omega \times\left(x_{0}-\varepsilon, x_{0}+\varepsilon\right)\right)$, which intersects $\Lambda_{\mathcal{P}}$ only once by (5.2)-hence, $\omega_{1}=\omega_{2}$.

Thus we can invert $\xi$, and $\left.\theta \circ \xi^{-1}\right|_{\xi\left(\Lambda_{\mathcal{P}}^{c}\right)}$ is a continuous function. Let $\widehat{\theta}: \Lambda_{\mathcal{P}} \rightarrow \mathbb{R}$ be its continuous extension to $\Lambda_{\mathcal{P}}$. To construct a homotopy $h_{\lambda}: \mathcal{S}_{\mathcal{P}} \rightarrow \mathcal{S}_{\mathcal{P}}, \lambda \in[0,1]$, supported on $\pi\left(\Lambda_{\mathcal{P}} \times\left[x_{0}-2 \varepsilon, x_{0}+2 \varepsilon\right]\right)$, fix a vector field $X$ supported on $\left[x_{0}-2 \varepsilon, x_{0}+2 \varepsilon\right]$ and such that $X=1$ on $\left[x_{0}-\varepsilon, x_{0}+\varepsilon\right]$. Denote by $X^{t}$ the flow of $X$. For $z \in \pi\left(\Lambda_{\mathcal{P}} \times\left(x_{0}-2 \varepsilon, x_{0}+2 \varepsilon\right)\right)$, set

$$
h_{\lambda}(z)=\pi\left(\omega, X^{\lambda\left[x_{0}-\widehat{\theta}(\omega)\right]} x\right)
$$

where $(\omega, x) \in \Lambda_{\mathcal{P}} \times\left(x_{0}-2 \varepsilon, x_{0}+2 \varepsilon\right)$ is the (unique) lift of $z$. Extend $h_{\lambda}$ by identity to the whole $\mathcal{S}_{\mathcal{P}}$. This definition assures that $h_{0}=\mathbf{I d}$ and $h_{1} \circ f\left(\Lambda_{\mathcal{P}}^{c}\right)=\pi \circ\left(\xi\left(\Lambda_{\mathcal{P}}^{c}\right) \times x_{0}\right)$. Moreover, $h_{\lambda}$ is a homeomorphism for each $\lambda \in[0,1]$ : it is manifestly surjective, and it is $1-1$ because it preserves the composants $\pi(\omega \times \mathbb{R})$ and solves an ODE on each of them. In this way, the isotopy given by $T^{-\lambda x_{0}} \circ h_{\lambda} \circ f, \lambda \in[0,1]$, deforms $f$ to $\bar{f}:=T^{-x_{0}} \circ h_{1} \circ f$ such that $\bar{f}\left(\Lambda_{\mathcal{P}}^{c}\right) \subset \Lambda_{\mathcal{P}}$, as required. 
STEP 2: Adjust $\bar{f}$ by translation so that the neutral element $e$ is a fixed point; namely, define

$$
\widehat{f}:=s_{\bar{f}(e)^{-1}} \circ \bar{f}
$$

so that $\widehat{f}(e)=e$.

FACT 5.4. $\widehat{f}\left(\Lambda_{\mathcal{P}}^{c}\right)$ is a cross section of $T^{t}$ with some constant return time $d \in \mathbb{N}$.

Proof. Fix $\omega_{0} \in \Lambda_{\mathcal{P}}^{c}$ and set $\alpha(t):=\widehat{f} \circ T^{t}\left(\omega_{0}\right)$ for $t \in \mathbb{R}$. By Fact 5.3, $\alpha(t)=T^{\tau(t)} \omega$ for some $\omega \in \Lambda_{\mathcal{P}}$ and $\tau: \mathbb{R} \rightarrow \mathbb{R}$ an increasing homeomorphism (since we assumed that $f$ preserved orientation). Because $\alpha(0), \alpha(c) \in$ $\widehat{f}\left(\Lambda_{\mathcal{P}}^{c}\right) \subset \Lambda_{\mathcal{P}}$, the winding number of the curve $\left.\mathrm{pr}_{0} \circ \alpha\right|_{[0, c]}$ into the zeroth circle is well defined; denote it by $d \in \mathbb{N}$. Since $c$ is the first return time of $\omega_{0}$ to $\Lambda_{\mathcal{P}}^{c}$ and $\widehat{f}$ is an orbit equivalence, $d$ is the first return time of $\widehat{f}\left(\omega_{0}\right)$ to $\widehat{f}\left(\Lambda_{\mathcal{P}}^{c}\right)$. The condition (5.3) assures that $d$ does not depend on $\omega_{0}$ because, as we vary $\omega_{0} \in \mathcal{S}_{\mathcal{P}}^{c}$, the different curves $\operatorname{pr}_{0} \circ \alpha(t)$ never depart from each other farther than by $1 / 4$, i.e. $\operatorname{dist}\left(\operatorname{pr}_{0} \circ \alpha(t), \operatorname{pr}_{0} \circ \widetilde{\alpha}(t)\right) \leq 1 / 4$ if $\widetilde{\alpha}(t):=\widehat{f} \circ T^{t}\left(\widetilde{\omega}_{0}\right)$ for some $\widetilde{\omega}_{0} \in \Lambda_{\mathcal{P}}^{c}$.

Set $\phi:=\left.\widehat{f}\right|_{\Lambda_{\mathcal{P}}^{c}}$. Observe that $\phi\left(\gamma^{c n}\right)=\gamma^{d n}$ for $n \in \mathbb{Z}$ because the first return to $\Lambda_{\mathcal{P}}^{c}$ corresponds via $\widehat{f}$ to the first return to $\widehat{f}\left(\Lambda_{\mathcal{P}}\right)$ after time $d$ (see Fact 5.4). Since $\overline{\left\{\gamma^{n}: n \in \mathbb{N}\right\}}=\Lambda_{\mathcal{P}}$, we conclude that

$$
\phi \circ \phi_{c}=\phi_{d}
$$

(Here $\phi_{k}(\omega)=\omega^{k}$, see Section 3.) In particular, $\widehat{f}\left(\Lambda_{\mathcal{P}}^{c}\right)=\Lambda_{\mathcal{P}}^{d}$.

FACT 5.5. The return times $c$ and $d$ are products of primes in $\mathcal{P}$, i.e. $c=c_{\mathcal{P}}$ and $d=d_{\mathcal{P}}$. Moreover, $c / d=c_{\mathcal{P}, \infty} / d_{\mathcal{P}, \infty}$.

Proof. From Fact 3.2 , the return time to $\Lambda_{\mathcal{P}}^{d}$ equals $\left[\Lambda_{\mathcal{P}}: \Lambda_{\mathcal{P}}^{d}\right]=d_{\mathcal{P}}$, which shows that $d=d_{\mathcal{P}}$. That $c=c_{\mathcal{P}}$ follows from the definition of $c$. For the "moreover" part, note that $\phi$ is $1-1$ so that $\left|\operatorname{ker}\left(\phi_{c}\right)\right|=\left|\operatorname{ker}\left(\phi_{d}\right)\right|$ by (5.4), which translates into $c_{\mathcal{P},+}=d_{\mathcal{P},+}$ via Fact 3.1. Hence, $c / d=c_{\mathcal{P}} / d_{\mathcal{P}}=$ $\left(c_{\mathcal{P},+} c_{\mathcal{P}, \infty}\right) /\left(d_{\mathcal{P},+} d_{\mathcal{P}, \infty}\right)=c_{\mathcal{P}, \infty} / d_{\mathcal{P}, \infty}$.

STEP 3: There is an isotopy of $\widehat{f}$ to the affine homomorphism $g_{\mathcal{C}_{\mathcal{P}, \infty} / d_{\mathcal{P}, \infty}}$ $:=g_{c_{\mathcal{P}, \infty}} \circ g_{d_{\mathcal{P}, \infty}}^{-1}$.

The idea is that (5.4) forces $\widehat{f}$ to agree with $g_{c / d}$ on $\Lambda_{\mathcal{P}}^{c}$, and this agreement can be easily spread onto the whole $\mathcal{S}_{\mathcal{P}}$ by "ironing out" the nonlinearities along the fibers $\omega \times \mathbb{R}$.

Construction. We only produce a homotopy and leave derivation of formulas for an isotopy as an exercise. Because $\widehat{f}\left(\Lambda_{\mathcal{P}}^{c}\right)=\Lambda_{\mathcal{P}}^{d}$ we can cut $\mathcal{S}_{\mathcal{P}}$ 
along the cross sections $\Lambda_{\mathcal{P}}^{c}$ and $\Lambda_{\mathcal{P}}^{d}$ and lift $g$ to $\widetilde{g}$ as in the diagram below:

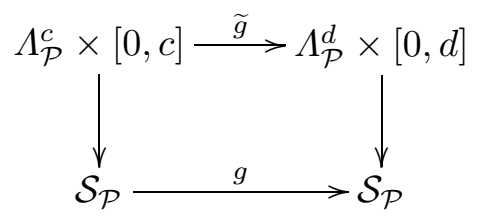

where the vertical arrows realize the boundary identifications $(\omega, c) \sim$ $\left(T^{c} \omega, 0\right)$ and $(\omega, d) \sim\left(T^{d} \omega, 0\right)$.

Since $\widehat{f}$ preserves the flow lines of $T,(5.4)$ implies that $\widetilde{g}\left(\omega^{c}, x\right):=$ $\left(\omega^{d}, h\left(\omega^{c}, x\right)\right),\left(\omega^{c}, x\right) \in \Lambda_{\mathcal{P}}^{c} \times[0, c]$, for some $h: \Lambda_{\mathcal{P}}^{c} \times[0, c] \rightarrow[0, d]$ that maps $\Lambda_{\mathcal{P}}^{c} \times\{0, c\}$ to $\{0, d\}$. (Note that $\omega^{d}$ is well defined in terms of $\omega^{c}$ because $\operatorname{ker}\left(\phi_{d}\right)=\operatorname{ker}\left(\phi_{c}\right)$ by (5.4).) We write a homotopy $\widetilde{g}_{\lambda}: \Lambda_{\mathcal{P}}^{c} \times[0, c] \rightarrow$ $\Lambda_{\mathcal{P}}^{d} \times[0, d], \lambda \in[0,1]$,

$$
\widetilde{g}_{\lambda}\left(\omega^{c}, x\right):=\left(\omega^{d},(d / c) \cdot x+(1-\lambda) \cdot\left(h\left(\omega^{c}, x\right)-(d / c) \cdot x\right)\right) .
$$

Observe that $\widetilde{g}$ respects the identifications on $\Lambda_{\mathcal{P}}^{c} \times\{0, c\}$ and $\Lambda_{\mathcal{P}}^{d} \times\{0, d\}$ so that it descends to a homotopy $\widehat{g}_{\lambda}: \mathcal{S}_{\mathcal{P}} \rightarrow \mathcal{S}_{\mathcal{P}}$. Clearly, $\widehat{g}_{0}=\widehat{f}$ and we claim that $\widehat{g}_{1}=g_{\mathcal{C}_{\mathcal{P}, \infty} / d_{\mathcal{P}, \infty}}$. Indeed, given $z \in \mathcal{S}_{\mathcal{P}}$, we take its lift $\left(\omega^{c}, x\right) \in$ $\Lambda_{\mathcal{P}}^{c} \times[0, c]$ and see that $c / d=c_{\mathcal{P}, \infty} / d_{\mathcal{P}, \infty}$ implies that

$$
\widetilde{g}_{1}\left(\omega^{c c_{\mathcal{P}, \infty}}, c_{\mathcal{P}, \infty} x\right)=\left(\omega^{d c_{\mathcal{P}, \infty}},(d / c) \cdot c_{\mathcal{P}, \infty} x\right)=\left(\omega^{c d_{\mathcal{P}, \infty}}, d_{\mathcal{P}, \infty} x\right),
$$

which shows that $\widehat{g}_{1}\left(z^{c_{\mathcal{P}, \infty}}\right)=z^{d_{\mathcal{P}, \infty}}$ for all $z \in \mathcal{S}_{\mathcal{P}}$, i.e. $\widehat{g}_{1}=g_{\mathcal{C}_{\mathcal{P}, \infty} / d_{\mathcal{P}, \infty}}$.

Conclusion of the proof of Theorem 1. The progression of the three steps above shows that if $f: \mathcal{S}_{\mathcal{P}} \rightarrow \mathcal{S}_{\mathcal{P}}$ is a homeomorphism, then $g_{\mathcal{P}_{\mathcal{P}, \infty} / d_{\mathcal{P}, \infty}}$ is isotopic to $s_{\omega^{-1}} \circ f$ in the orientation preserving case or to $s_{\omega^{-1}} \circ r \circ f$ in the orientation reversing case. (Here $\omega:=\bar{f}(e)$.) Thus $f$ is isotopic to $g_{\mathcal{C}_{\mathcal{P}, \infty} / d_{\mathcal{P}, \infty}, \omega}$ or to $r \circ g_{\mathcal{C}_{\mathcal{P}, \infty} / d_{\mathcal{P}, \infty, \omega}}$.

6. General form of homeomorphism. The results of the previous two sections yield a very explicit description of homeomorphisms of $\mathcal{S}_{\mathcal{P}}$ :

Corollary 6.1. If $f: \mathcal{S}_{\mathcal{P}} \rightarrow \mathcal{S}_{\mathcal{P}}$ is an orientation preserving homeomorphism, then $f$ has a lift $F: \Lambda_{\mathcal{P}} \times \mathbb{R} \rightarrow \Lambda_{\mathcal{P}} \times \mathbb{R}$ of the form

$$
F:(\omega, x) \mapsto\left(\sigma \cdot \phi_{a / b}(\omega), \frac{a\left(x+I_{b}(\omega)\right)}{b}+\delta(\omega, x)\right)
$$

where $a$ and $b$ are co-prime $\mathcal{P}$-recurrent, $\sigma \in \Lambda_{\mathcal{P}}$, and $\delta: \Lambda_{\mathcal{P}} \times \mathbb{R} \rightarrow \mathbb{R}$ is a bounded continuous function invariant under the deck map: $\delta \circ D=\delta$, i.e. $\delta(\gamma \cdot \omega, x-1)=\delta(\omega, x)$ for all $(\omega, x) \in \Lambda_{\mathcal{P}} \times \mathbb{R}$. If $f: \mathcal{S}_{\mathcal{P}} \rightarrow \mathcal{S}_{\mathcal{P}}$ is orientation reversing, then it has a lift of the form $R \circ F$ (where, recall, $R(\omega, x)=$ $\left.\left(\omega^{-1},-x\right)\right)$. Moreover, the numbers $a$ and $b$ are uniquely determined by the homotopy type of $f$. 
Observe that $F \circ D=D^{\kappa(\cdot)} \circ F$ where $\kappa(\omega, x)=0$ if $I_{b}(\omega)<b-1$ and $\kappa(\omega, x)=a$ if $I_{b}(\omega)=b-1$ (cf. the proof of Lemma 4.1).

Proof of Corollary 6.1. Theorem 1 supplies a homotopy $f_{\lambda}: \mathcal{S}_{\mathcal{P}} \rightarrow \mathcal{S}_{\mathcal{P}}$, $\lambda \in[0,1]$, that connects $f=f_{1}$ to an affine homeomorphism $g=f_{0}$. Let $F_{\lambda}: \Lambda_{\mathcal{P}} \times \mathbb{R} \rightarrow \Lambda_{\mathcal{P}} \times \mathbb{R}, \lambda \in[0,1]$, be a lift of this homotopy such that $F_{0}$ is the lift $G_{a / b, \sigma}$ of the affine homeomorphism (see Definition 4.1). Recall that, since $\pi$ is a covering, $F_{\lambda}(\omega, x)$ is obtained by unique path lifting of the path $[0,1] \ni \lambda \mapsto f_{\lambda}(z), z=\pi(\omega, x)$, starting at a point $(\mu, y):=$ $G_{a / b, \sigma}(\omega, x)$. Total disconnectedness of $\Lambda_{\mathcal{P}}$ forces the lifted path to have a constant projection onto $\Lambda_{\mathcal{P}}$, i.e. to be of the form $(\mu, y+\widetilde{\delta}(\omega, x, \lambda))$ for some continuous $\widetilde{\delta}: \Lambda_{\mathcal{P}} \times \mathbb{R} \times[0,1] \rightarrow \mathbb{R}$. Thus the assertion holds with $\delta(\omega, x):=\widetilde{\delta}(\omega, x, 1)$. The equivariance of $\delta$ is immediate from the uniqueness of path lifting. The uniqueness of $a$ and $b$ is a consequence of Lemma 4.2.

7. Entropy (proof of Theorem 2). To determine the entropy of an arbitrary homeomorphism $f: \mathcal{S}_{\mathcal{P}} \rightarrow \mathcal{S}_{\mathcal{P}}$ and prove Theorem 2, we shall pass to the lift $F$ of $f$ given by (6.1), compute the entropies generated by $F$ on the base $\Lambda_{\mathcal{P}}$ and in the individual fibers $\omega \times \mathbb{R}$, and apply a version of Bowen's formula for the entropy of skew products (as shown in the appendix). The references [4] and [21] may be consulted for the definition of topological entropy. All the entropies are computed with respect to the metrics on $\Lambda_{\mathcal{P}}$, $\mathcal{S}_{\mathcal{P}}$, and $\Lambda_{\mathcal{P}} \times \mathbb{R}$ constructed in Section 2 .

LEMMA 7.3. For a map $F$ as in Corollary 6.1 , a compact segment $I \subset \mathbb{R}$, and $\omega \in \Lambda_{\mathcal{P}}$, the entropy of the compact set $\omega \times I$ with respect to $F$ and the metric $d_{\Lambda_{\mathcal{P}} \times \mathbb{R}}$ satisfies

$$
h(F, \omega \times I) \leq \max \{0, \log (a / b)\} .
$$

The lemma hinges on the well known basic fact that, given two partitions of a line segment: one into $p$ subsegments $\left\{I_{i}\right\}$ and another into $q$ subsegments $\left\{J_{j}\right\}$, their refinement $\left\{I_{i}\right\} \vee\left\{J_{j}\right\}:=\left\{I_{i} \cap J_{j}\right\}_{i, j}$ can have at most $p+q-1$ non-empty elements. This indicates that the entropy is produced solely by exponential stretching, which is controlled by $a / b$. (Thus, in fact, $h_{d_{\Lambda_{\mathcal{P}} \times \mathbb{R}}}(F, \omega \times \mathbb{R})=\max \{0, \log (a / b)\}$.)

Proof of Lemma 7.3. Fix arbitrary $\varepsilon, \lambda>0$ such that $\lambda>\max \{1,|a / b|\}$. Set $\omega_{k}:=\operatorname{pr}_{\Lambda_{\mathcal{P}}} \circ F^{k}(\omega \times I)$ and $I_{k}:=\operatorname{pr}_{\mathbb{R}} \circ F^{k}(\omega \times I), k \geq 0$. It is easy to see from (6.1) that there is $C>0$ such that $\left|I_{k}\right| \leq C \lambda^{k}$ for $k \geq 0$. Let $\mathcal{A}_{k}$ be a partition of $\omega_{k} \times I_{k}$ into closed segments of equal length $\varrho \in[\varepsilon / 2, \varepsilon]$ so that the cardinality $\# \mathcal{A}_{k} \leq 2 C \lambda^{k} / \varepsilon$. The partition $\mathcal{A}^{n}:=\mathcal{A}_{0} \vee F^{-1} \mathcal{A}_{1} \vee$ $\ldots \vee F^{-n+1} \mathcal{A}_{n-1}$ has cardinality $\# \mathcal{A}^{n} \leq(2 C / \varepsilon) \cdot\left(1+\lambda+\ldots+\lambda^{n}+n\right)$. Since any selector set of $\mathcal{A}^{n}$ is an $(\varepsilon, n)$-spanning set in $\omega \times I$, we obtain 
$h_{d_{\Lambda_{\mathcal{P}} \times \mathbb{R}}}(F, \omega \times I) \leq \log \lambda$ after letting $n \rightarrow \infty$ and then $\varepsilon \rightarrow \infty$. We are done by the arbitrariness of $\lambda$.

Lemma 7.4. For $a, b \in \mathbb{N}$ co-prime with $b \mathcal{P}$-recurrent, the topological entropy of the map $\sigma \cdot \phi_{a / b}: \Lambda_{\mathcal{P}} \rightarrow \Lambda_{\mathcal{P}}$ is

$$
h\left(\sigma \cdot \phi_{a / b}\right)=\log b .
$$

Proof. It is more convenient to argue for $\psi:=w+\psi_{a / b}: \lim _{\longleftarrow} \mathbb{Z}_{p_{1}, \ldots, p_{k}} \rightarrow$ $\underset{\lim }{\mathbb{Z}_{p_{1}, \ldots, p_{k}}}$ that is conjugate to $\sigma \cdot \phi_{a / b}$ (as explained in Remark 4.1). Let $\left.\overleftarrow{\left(k_{n}\right.}\right)_{n=1}^{\infty}$ be a non-decreasing sequence such that $p_{1} \ldots p_{k_{n}}$ is divisible by $b^{n}, n \in \mathbb{N}$; and set $\varepsilon:=\exp \left(-k_{1}\right)$. We claim that if $s, \widetilde{s} \in \lim \mathbb{Z}_{p_{1}, \ldots, p_{k}}$ are such that $s_{k} \not \equiv \widetilde{s}_{k}\left(\bmod b^{n}\right)$ for some $k \geq k_{n}$, then $s$ and $\widetilde{s}$ are $(n, \varepsilon)$ separated. This yields $h(\psi) \geq \log b$ because, by using the natural embedding $\kappa: \mathbb{N} \rightarrow \lim _{p_{1}, \ldots, p_{k}}$ such that $\left(\kappa(n)_{k}\right)_{k=1}^{\infty}:=\left(n\left(\bmod p_{1} \ldots p_{k}\right)\right)_{k=1}^{\infty}$, we exhibit an $(n, \varepsilon)$-separated set $S_{n}:=\kappa\left(\left\{0, \ldots, b^{n}-1\right\}\right) \subset \lim _{\longleftarrow} \mathbb{Z}_{p_{1}, \ldots, p_{k}}$ and $\# S_{n}=b^{n}$.

We verify the claim by induction on $n$. For $n=1$, the $\mathcal{P}$-adic expansions $\left[d_{k}\right]$ and $\left[\widetilde{d}_{k}\right]$ of $s$ and $\widetilde{s}$ (see (2.1)) must differ on some digit $d_{k_{*}} \neq \widetilde{d}_{k_{*}}$ for $k_{*} \leq k_{1}$ since otherwise $\widetilde{s}_{k}-s_{k}$ would be divisible by $b$. Thus $\mathrm{d}_{\Lambda_{\mathcal{P}}}(s, \widetilde{s}) \geq \varepsilon$ by the definitions of $\varepsilon$ and the distance $\mathrm{d}_{\Lambda_{\mathcal{P}}}$ (Section 2). Now, for the induction step, assume that the claim holds for $n-1$ and that $s_{k} \not \equiv \widetilde{s}_{k}\left(\bmod b^{n}\right)$. Unless $\mathrm{d}_{\Lambda_{\mathcal{P}}}(s, \widetilde{s}) \geq \varepsilon$ (when there is nothing to show), we have $s_{k} \equiv \widetilde{s}_{k}(\bmod b)$ for $k \geq k_{1}$ by what we have already shown. Hence, $s_{k} \div b \not \equiv \widetilde{s}_{k} \div b\left(\bmod b^{n-1}\right)$. Since $a$ is co-prime to $b$, it follows that

$$
\psi(s)_{k}=w_{k}+a\left(s_{k} \div b\right) \not \equiv w_{k}+a\left(\widetilde{s}_{k} \div b\right)=\psi(\widetilde{s})_{k}\left(\bmod b^{n-1}\right),
$$

and we conclude that $\psi(s)$ and $\psi(s)$ are $(n-1, \varepsilon)$-separated by the induction hypothesis. This makes $s$ and $\widetilde{s}(n, \varepsilon)$-separated.

To prove $h(\psi) \leq \log b$, it suffices to show that if $s, \widetilde{s} \in \varliminf_{\lim _{1}} \mathbb{Z}_{p_{1}, \ldots, p_{k}}$ are such that $s_{k} \equiv \widetilde{s}_{k}\left(\bmod p_{1} \ldots p_{k_{1}} b^{n}\right)$ for some $k>k_{1}$ large enough so that $p_{1} \ldots p_{k_{1}} b^{n}$ divides $p_{1} \ldots p_{k}$, then $s$ and $\widetilde{s}$ are $(n, \varepsilon)$-close. Indeed, $\widetilde{S}_{n}:=$ $\kappa\left(\left\{0, \ldots, p_{1} \ldots p_{k_{1}} b^{n}-1\right\}\right)$ is then an $(n, \varepsilon)$-spanning set with cardinality $p_{1} \ldots p_{k_{1}} b^{n}$, and $\varepsilon$ can be made arbitrarily small by increasing $k_{1}$. Thus, suppose that $s_{k} \equiv \widetilde{s}_{k}\left(\bmod p_{1} \ldots p_{k_{1}} b^{n}\right)$. Then $s_{k} \equiv \widetilde{s}_{k}\left(\bmod p_{1} \ldots p_{k_{1}}\right)$ and so $\mathrm{d}_{\Lambda_{\mathcal{P}}}(s, \widetilde{s}) \leq \varepsilon$. Also, $s_{k} \div b \equiv \widetilde{s}_{k} \div b\left(\bmod p_{1} \ldots p_{k_{1}} b^{n-1}\right)$ so that

$$
\psi(s)_{k}=w_{k}+a\left(s_{k} \div b\right) \equiv w_{k}+a\left(\widetilde{s}_{k} \div b\right)=\psi(\widetilde{s})_{k}\left(\bmod p_{1} \ldots p_{k_{1}} b^{n-1}\right),
$$

which shows that $\mathrm{d}_{\Lambda_{\mathcal{P}}}(\psi(s), \psi(\widetilde{s})) \leq \varepsilon$. By repeating the argument, $\psi^{i}(s)_{k} \equiv$ $\psi^{i}(\widetilde{s})_{k}\left(\bmod p_{1} \ldots p_{k_{1}} b^{n-i}\right)$ so that $\mathrm{d}_{\Lambda_{\mathcal{P}}}\left(\psi^{i}(s), \psi^{i}(\widetilde{s})\right) \leq \varepsilon$ for $i=0, \ldots$, $n-1$.

Our Theorem 2 amounts to the following proposition. 
Proposition 7.1. The topological entropy $h(f)$ of a homeomorphism $f$ : $\mathcal{S}_{\mathcal{P}} \rightarrow \mathcal{S}_{\mathcal{P}}$ is given by

$$
h(f)=\max \{0, \log (a / b)\}+\log b=\log \max \{a, b\}
$$

where $a$ and $b$ are as in Corollary 6.1.

Proof. It suffices to argue for $f$ that is orientation preserving (otherwise, consider $\left.f^{2}\right)$. First, note that $h(f)=h(F, K)$ for $K:=\Lambda_{\mathcal{P}} \times[-M, M]$ where $M \geq 1$ (cf. Theorem 8.12 of [21]). To see that, fix $1 / 4>r>0$ so that $\left.\pi\right|_{B(p, r)}: B(p, r) \rightarrow B(\pi(p), r)$ is a surjective isometry for all $p \in \Lambda_{\mathcal{P}} \times \mathbb{R}$ (where $B(p, r)=\{z: d(z, p)<r\}$ ). Consider any $\varepsilon \in(0, r)$ small enough that $d(F(p), F(q))<r$ if $d(p, q)<\varepsilon$. One can verify that if $E$ is $(n, \varepsilon)-$ spanning for $\mathcal{S}_{\mathcal{P}}$, then $\widetilde{E}:=\pi^{-1}(E) \cap\{p \in K: d(p, K) \leq \varepsilon\}$ is $(n, \varepsilon)$ spanning for $K$; and $\# \widetilde{E} \leq(2 M+1) \# E$, which implies $h(F, K) \leq h(f)$. Also, if $\widetilde{E}$ is $(n, \varepsilon)$-spanning for $K$, then $E:=\pi(\widetilde{E})$ is $(n, \varepsilon)$-spanning for $\mathcal{S}_{\mathcal{P}}$, which implies $h(f) \leq h(F, K)$.

Since passing to $f^{-1}$ interchanges the roles of $a$ and $b$ (cf. (4.5)) and $h(f)=h\left(f^{-1}\right)$, we may assume that $a / b \leq 1$. Suppose first that $a / b<1$. For some large $M>1, K=\Lambda_{\mathcal{P}} \times[-M, M]$ is invariant under $F, F(K) \subset K$. The projection $K \rightarrow \Lambda_{\mathcal{P}}$ factors $\left.F\right|_{K}$ to $\sigma \cdot \phi_{a / b}: \Lambda_{\mathcal{P}} \rightarrow \Lambda_{\mathcal{P}}$. Since entropy cannot increase under factoring, $h\left(\left.F\right|_{K}\right) \geq h\left(\sigma \cdot \phi_{a / b}\right)=\log b$, where we also used Lemma 7.4. Bowen's theorem (Theorem 17 of [4], cf. Theorem 5), combined with Lemma 7.3, yields

$$
h\left(\left.F\right|_{K}\right) \leq h\left(\sigma \cdot \phi_{a / b}\right)+\sup _{\omega \in \Lambda_{\mathcal{P}}} h(F, \omega \times[-M, M])=\log b+0 .
$$

Thus $h(f)=h(F, K)=h\left(\left.F\right|_{K}\right)=\log b$, and we are done for $a / b \neq 1$.

Suppose now $a / b=1$, that is, $a=b=1$. One easily checks that Theorem 5 (formulated and shown in the appendix) can be applied to $X:=$ $\Lambda_{\mathcal{P}} \times \mathbb{R}$ with $G:=\mathbb{Z}$ acting as described in Section 2 and $K:=\Lambda_{\mathcal{P}} \times[-1,1]$, $Y:=\Lambda_{\mathcal{P}}, T:=F$, and $S:=\sigma \cdot \phi_{1}$. Therefore, we obtain

$$
h(f)=h(F, K) \leq h\left(\sigma \cdot \phi_{1}\right)+\sup _{\omega \in \Lambda_{\mathcal{P}}} h(F, \omega \times[-1,1])=\log 1+0=0 .
$$

8. Global shadowing (proof of Theorem 3). In this section, we prove Theorem 3, that is, we fix a homeomorphism $f: \mathcal{S}_{\mathcal{P}} \rightarrow \mathcal{S}_{\mathcal{P}}$ with winding ratio $a / b \neq 1$ and show that $f$ is semi-conjugate to the affine homeomorphism $g: \mathcal{S}_{\mathcal{P}} \rightarrow \mathcal{S}_{\mathcal{P}}$ isotopic to $f$ provided by Theorem 1 (i.e. we construct a continuous surjective map $h: \mathcal{S}_{\mathcal{P}} \rightarrow G$ such that $\left.h \circ f=g \circ h\right)$. As before, we argue mostly at the level of lifts $F, G: \Lambda_{\mathcal{P}} \times \mathbb{R} \rightarrow \Lambda_{\mathcal{P}} \times \mathbb{R}$ of $f$ and $g$. By Corollary 6.1, these exist as a pair of equivariantly homotopic maps of the form

$$
F(\omega, x)=(\phi(\omega), \lambda x+\delta(\omega, x)), \quad G(\omega, x)=(\phi(\omega), \lambda x+\Delta(\omega)),
$$


where $\lambda= \pm a / b$ according as $f$ is orientation preserving or not, and $\Delta(\cdot)=$ $a / b \cdot I_{b}(\cdot)$. Note that we may assume that $|\lambda|>1$ by passing to $f^{-1}$ if necessary. We also fix $\mu \in(1,|\lambda|)$ and set $C:=(\sup |\delta|+\sup |\Delta|) /(|\lambda|-\mu)$ $<\infty$.

Fix (for a moment) points $(\omega, x),(\omega, y) \in \Lambda_{\mathcal{P}} \times \mathbb{R}$ together with the sequences

$$
x_{n}:=\operatorname{pr}_{\mathbb{R}}\left(F^{n}(\omega, x)\right) \quad \text { and } \quad y_{n}:=\operatorname{pr}_{\mathbb{R}}\left(G^{n}(\omega, y)\right), \quad n \geq 0 .
$$

Adopting Katok's notion of global shadowing and by emulating [9], we say that $(\omega, x)$ is $C$-shadowed by $(\omega, y)$, and write $(\omega, x) \rightsquigarrow(\omega, y)$, if $\left|x_{n}-y_{n}\right| \leq$ $C$ for all $n \geq 0$. The following proposition shows that $H: \Lambda_{\mathcal{P}} \times \mathbb{R} \rightarrow \Lambda_{\mathcal{P}} \times \mathbb{R}$ that associates to $(\omega, x)$ its $C$-shadow $(\omega, y)$ is well defined and factors to the required semi-conjugacy $h$.

Proposition 8.2. (i) For any $(\omega, x) \in \Lambda_{\mathcal{P}} \times \mathbb{R}$, there is a unique $(\omega, y)$ that $C$-shadows $(\omega, x)$, i.e. $(\omega, x) \rightsquigarrow(\omega, y)$.

(ii) $H: \Lambda_{\mathcal{P}} \times \mathbb{R} \rightarrow \Lambda_{\mathcal{P}} \times \mathbb{R},(\omega, x) \rightsquigarrow(\omega, y)$, is continuous.

(iii) $D \circ H=H \circ D$ so that $H$ factors to a map $h: \mathcal{S}_{\mathcal{P}} \rightarrow \mathcal{S}_{\mathcal{P}}, \pi \circ H=h \circ \pi$.

(iv) $H \circ F=G \circ H$ and $h \circ f=g \circ h$.

(v) $h$ is surjective.

Proof. (i) Fix $(\omega, x) \in \Lambda_{\mathcal{P}} \times \mathbb{R}$. Let $\varrho=1$ if $f$ is orientation preserving and $\varrho=-1$ otherwise (so that $\lambda=\varrho \cdot a / b$ ). Consider the open sets

$$
\begin{aligned}
& E^{-}:=\left\{y \in \mathbb{R}: \varrho^{n} y_{n}<\varrho^{n} x_{n}-C \text { for some } n \geq 0\right\}, \\
& E^{+}:=\left\{y \in \mathbb{R}: \varrho^{n} y_{n}>\varrho^{n} x_{n}+C \text { for some } n \geq 0\right\} .
\end{aligned}
$$

We have to show that $\mathbb{R} \backslash\left(E^{-} \cup E^{+}\right)$is a single point as this is the set of $y$ 's for which $(\omega, x) \rightsquigarrow(\omega, y)$. The choice of the constant $C$ guarantees that, given $k \geq 0$,

$$
\begin{array}{lll}
\varrho^{k} y_{k}>\varrho^{k} x_{k}+C & \text { implies } & \varrho^{n} y_{n}>\varrho^{n} x_{n}+\mu^{n-k} C \text { for } n \geq k, \\
\varrho^{k} y_{k}<\varrho^{k} x_{k}-C & \text { implies } & \varrho^{n} y_{n}<\varrho^{n} x_{n}-\mu^{n-k} C \text { for } n \geq k .
\end{array}
$$

Hence $E^{-}$and $E^{+}$are disjoint. The monotonicity of the maps $x \mapsto \lambda x+$ $\delta(\omega, x)$ and $x \mapsto \lambda x+\Delta(\omega)$ implies that $E^{-}$contains with each $y$ all points in $(-\infty, y]$, i.e. $E^{-}$is an infinite segment $E^{-}=\left(-\infty, y^{-}\right)$for some $y^{-} \in \mathbb{R}$. Similarly, $E^{+}=\left(y^{+}, \infty\right)$ for some $y^{+} \in \mathbb{R}$. Since $y^{-}, y^{+} \notin E^{-} \cup E^{+}$, we have $(\omega, x) \rightsquigarrow\left(\omega, y^{-}\right)$and $(\omega, x) \rightsquigarrow\left(\omega, y^{+}\right)$; however, $y_{n}^{+}-y_{n}^{-}=\lambda^{n}\left(y^{+}-y^{-}\right)$ and thus necessarily $y^{+}=y^{-}$, which ends the proof of (i).

(ii) Fix an arbitrary $\varepsilon>0$. Pick $n \in \mathbb{N}$ so that $3 C /|\lambda|^{n} \leq \varepsilon$. Consider $(\omega, x),(\widetilde{\omega}, \widetilde{x}) \in \Lambda_{\mathcal{P}} \times \mathbb{R}$. Take $\delta>0$ such that $d_{\Lambda_{\mathcal{P}} \times \mathbb{R}}((\omega, x),(\widetilde{\omega}, \widetilde{x}))<\delta$ forces $\left|\widetilde{x}_{k}-x_{k}\right| \leq C$ and $\Delta\left(\phi^{k}(\omega)\right)=\Delta\left(\phi^{k}(\widetilde{\omega})\right)$ for $k=0, \ldots, n$; note that $\Delta$ is locally constant. For $(\omega, y):=H(\omega, x)$ and $(\widetilde{\omega}, \widetilde{y}):=H(\widetilde{\omega}, \widetilde{x})$, we see 
then that

$$
\left|\widetilde{y}_{n}-y_{n}\right| \leq\left|\widetilde{y}_{n}-\widetilde{x}_{n}\right|+\left|\widetilde{x}_{n}-x_{n}\right|+\left|y_{n}-x_{n}\right| \leq 3 C
$$

and

$$
\left|\widetilde{y}_{n}-y_{n}\right|=|\lambda|^{n}|\widetilde{y}-y|,
$$

which combine to yield $|\widetilde{y}-y| \leq 3 C /|\lambda|^{n} \leq \varepsilon$. This establishes continuity of $H$.

(iii) Since $F$ is a lift, for all $(\omega, x) \in \Lambda_{\mathcal{P}} \times \mathbb{R}$ we have $F \circ D(\omega, x)=$ $D^{k(\omega)} \circ F$, where $k: \Lambda_{\mathcal{P}} \rightarrow \mathbb{Z}$. Because $G$ is equivariantly homotopic to $F$, unique path lifting forces that $G \circ D(\omega, x)=D^{k(\omega)} \circ G$ with the same $k$. It follows that if $(\omega, x) \rightsquigarrow(\omega, y)$ then $D(\omega, x) \rightsquigarrow D(\omega, y)$. This establishes equivariance of $H$ and secures the existence of the factor $h$.

(iv) is a simple consequence of the definition of $C$-shadowing.

(v) Fix $(\omega, y) \in \Lambda_{\mathcal{P}} \times \mathbb{R}$. We can reverse the roles of $f$ and $g$ in the proof of part (i) and conclude that the orbit of $(\omega, y)$ under $G$ is $C$-shadowed by the orbit under $F$ of some $(\omega, x) \in \Lambda_{\mathcal{P}} \times \mathbb{R}$. (Uniqueness of $(\omega, x)$ may be lost due to non-linearities in $F$, cf. Remark 8.2 below.)

REMARK 8.2. The fibers of the semi-conjugacy $h$ are arcs of the form $h^{-1}(z)=\pi\left(\omega \times\left(x^{-}, x^{+}\right)\right)$. In particular, $h$ is guaranteed to be $1-1$, and thus a conjugacy if " $f$ is $C^{1}$-close enough to $g$ " so that $F$ expands the fibers. This happens for example when $x \mapsto \lambda x+\delta(\omega, x)$ has Lipschitz inverse with a constant $L<1$ that is uniform in $\omega$.

REMARK 8.3. Because $h^{-1}(z)$ 's are arcs, the fiber entropy is zero in Bowen's Theorem 17 of [4]; and we conclude $h(f)=h(g)$. This leads to an alternative proof of Theorem 2 for $f$ with winding ratio $a / b \neq 1$.

9. Knaster continua. Knaster continua can be realized by identifying each point of a solenoid with its inverse, i.e. we have $\mathbb{Z}_{2}$ acting via the involution $r: \mathcal{S}_{\mathcal{P}} \rightarrow \mathcal{S}_{\mathcal{P}}, r: z \mapsto z^{-1}$, and the $\mathcal{P}$-adic Knaster continuum is

$$
\mathcal{K}_{\mathcal{P}}:=\mathcal{S}_{\mathcal{P}} / \mathbb{Z}_{2} \text {. }
$$

We denote by $\mu: \mathcal{S}_{\mathcal{P}} \rightarrow \mathcal{K}_{\mathcal{P}}$ the natural projection. Observe that the restriction to $\mathcal{S}_{\mathcal{P}}$ of the product of the circle flattening maps, $\prod_{k=0}^{\infty} \mathbb{S} \rightarrow$ $\prod_{k=0}^{\infty}[-1,1]$ where $\left(e^{i \theta_{k}}\right)_{k=0}^{\infty} \mapsto\left(\cos \theta_{k}\right)_{k=0}^{\infty}$, establishes a homeomorphism of $\mathcal{K}_{\mathcal{P}}$ and the inverse limit of interval maps

$$
\mathcal{K}_{\mathcal{P}} \cong \varliminf_{\lim }\left(P_{p_{k}}:[-1,1] \rightarrow[-1,1]\right)
$$

where $P_{p}(\cos \theta)=\cos ^{p} \theta$ is the Chebyshev polynomial and has exactly $p$ monotonic laps each of which is surjective. One can now verify that the $\mathcal{K}_{\mathcal{P}}$ thus defined is homeomorphic to the Knaster continuum pictured in the introduction. 
The projection $\mu$ fails to be a cover at the fixed points of $r$, and it is easy to verify that we have two cases:

Even CASE: $\mathcal{P}$ contains infinitely many 2 's - then the neutral element $e$ is the only fixed point of $r$.

OdD CASE: $\mathcal{P}$ contains only finitely many 2's - then, besides $e$, there is exactly one more fixed point $e^{*}=(1, \ldots, 1,-1,-1,-1, \ldots)$ where the number of the leading 1's equals the maximal $k$ for which $p_{k}=2$ (or there are no 1 's if all $p_{k}$ 's are odd). In the "odd case", $e^{*} \cdot e^{*}=e$; and the two points $e$ and $e^{*}$ are interchanged by the translation $s_{e}^{*}: z \mapsto e^{*} \cdot z$, which factors to an involution $\widehat{s}: \mathcal{K}_{\mathcal{P}} \rightarrow \mathcal{K}_{\mathcal{P}}$.

We distinguish a class of standard homeomorphisms of $\mathcal{K}_{\mathcal{P}}$ (cf. [3]) that are the factors (through $\mu$ ) of the affine homeomorphisms of $\mathcal{S}_{\mathcal{P}}$. Since $g_{a / b, \sigma} \circ$ $r=r \circ g_{a / b, r(\sigma)}$, an affine homeomorphism $g_{a / b, \sigma}$ commutes with $r$ iff $r(\sigma)$ $=\sigma$, i.e. $\sigma \in\left\{e, e^{*}\right\}$. The standard homeomorphisms come then in two kinds: those fixing $\mu(e)$,

$$
\widehat{g}_{a / b,+}: \mathcal{K}_{\mathcal{P}} \rightarrow \mathcal{K}_{\mathcal{P}}, \quad \mu \circ g_{a / b, e}=\widehat{g}_{a / b,+} \circ \mu,
$$

and those interchanging $e$ and $e^{*}$,

$$
\widehat{g}_{a / b,-}: \mathcal{K}_{\mathcal{P}} \rightarrow \mathcal{K}_{\mathcal{P}}, \quad \mu \circ g_{a / b, e^{*}}=\widehat{g}_{a / b,-} \circ \mu ;
$$

the latter are well defined only in the "odd case". As before, $a, b$ run over pairs of $\mathcal{P}$-recurrent co-prime natural numbers. Clearly, $\widehat{g}_{a / b,-}=\widehat{s} \circ \widehat{g}_{a / b,+}$.

ThEOREM 4. If $\widehat{f}: \mathcal{K}_{\mathcal{P}} \rightarrow \mathcal{K}_{\mathcal{P}}$ is a homeomorphism, then

(i) there are $\mathcal{P}$-recurrent and co-prime $a, b \in \mathbb{N}$ such that $\widehat{f}$ is isotopic to a standard homeomorphism $g, g=\widehat{g}_{a / b,-}$ or $g=\widehat{g}_{a / b,+}$;

(ii) $\widehat{f}$ is semi-conjugate to $g$ provided $a / b \neq 1$;

(iii) $\widehat{f}$ has topological entropy $h(\widehat{f})=h(g)=\log \max \{a, b\}$.

We shall reduce the theorem to our results for $\mathcal{S}_{\mathcal{P}}$ via the following proposition.

Proposition 9.3. For any homeomorphism $\widehat{f}: \mathcal{K}_{\mathcal{P}} \rightarrow \mathcal{K}_{\mathcal{P}}$, there exists a unique orientation preserving homeomorphism $f: \mathcal{S}_{\mathcal{P}} \rightarrow \mathcal{S}_{\mathcal{P}}$ that is a lift of $\widehat{f}, \mu \circ f=\widehat{f} \circ \mu$.

It follows that $r \circ f$ is the only other lift of $\widehat{f}$. Before starting the proof, we define a metric $d_{\mathcal{K}_{\mathcal{P}}}$ on $\mathcal{K}_{\mathcal{P}}$ by $d_{\mathcal{K}_{\mathcal{P}}}(u, v):=\min \left\{d_{\mathcal{S}_{\mathcal{P}}}(w, z): \mu(w)=u\right.$, $\left.\mu(z)=v, w, z \in \mathcal{S}_{\mathcal{P}}\right\}$ for $u, v \in \mathcal{K}_{\mathcal{P}}$. We mention that, by using the $\mathcal{P}$-adic representation of $\Lambda_{\mathcal{P}}$, it is easy to verify that $\omega \mapsto \omega^{-1}$ is an isometry of $\Lambda_{\mathcal{P}}$ and that $\mathbb{Z}_{2}$ acts on $\mathcal{S}_{\mathcal{P}}$ isometrically so that $\mu$ is a local isometry at all points except $e$ and $e^{*}$. We will use the following lemma which expresses the idea that the points $z$ and $z^{-1}$ in $\mathcal{S}_{\mathcal{P}}$ can be distinguished by looking at the "orientation" of their composants projected to $\mathcal{K}_{\mathcal{P}}$. 
Lemma 9.5. There exists $\varrho>0$ such that if $\varepsilon<\varrho$, and $\tau:[0, \infty) \rightarrow$ $[0, \infty)$ is a homeomorphism, and

$$
d_{\mathcal{K}_{\mathcal{P}}}\left(\mu\left(T^{t} z\right), \mu\left(T^{-\tau(t)} z\right)\right)<\varepsilon \quad \text { for all } t \geq 0,
$$

then

$$
d_{\mathcal{S}_{\mathcal{P}}}\left(z, z^{-1}\right)<2 \varepsilon .
$$

Proof. Since $\pi: \Lambda_{\mathcal{P}} \times \mathbb{R} \rightarrow \mathcal{S}_{\mathcal{P}}$ is a local isometry, there is $\varrho>0$ such that $d_{\mathcal{S}_{\mathcal{P}}}\left(T^{t} z, T^{-\tau(t)} z\right)=t+\tau(t)$ for $t, \tau(t) \in[0, \varrho]$. Our hypothesis,

$$
\begin{aligned}
& d_{\mathcal{K}_{\mathcal{P}}}\left(\mu\left(T^{t} z\right), \mu\left(T^{-\tau(t)} z\right)\right) \\
& \quad=\min \left\{d_{\mathcal{S}_{\mathcal{P}}}\left(T^{t} z, T^{-\tau(t)} z\right), d_{\mathcal{S}_{\mathcal{P}}}\left(T^{t} z,\left(T^{-\tau(t)} z\right)^{-1}\right)\right\}<\varepsilon<\varrho,
\end{aligned}
$$

applied at the instance when $t+\tau(t)=\varepsilon$, yields $d_{\mathcal{K}_{\mathcal{P}}}\left(\mu\left(T^{t} z\right), \mu\left(T^{-\tau(t)} z\right)\right)$ $=d_{\mathcal{S}_{\mathcal{P}}}\left(T^{t} z,\left(T^{-\tau(t)} z\right)^{-1}\right)<\varepsilon$. By the triangle inequality,

$$
\begin{aligned}
d_{\mathcal{S}_{\mathcal{P}}}\left(z, z^{-1}\right) & \leq d_{\mathcal{S}_{\mathcal{P}}}\left(z, T^{t} z\right)+d_{\mathcal{S}_{\mathcal{P}}}\left(T^{t} z, T^{\tau(t)}\left(z^{-1}\right)\right)+d_{\mathcal{S}_{\mathcal{P}}}\left(T^{\tau(t)}\left(z^{-1}\right), z^{-1}\right) \\
& <t+\varepsilon+\tau(t)=2 \varepsilon .
\end{aligned}
$$

Under the projection $\eta:=\mu \circ \pi: \Lambda_{\mathcal{P}} \times \mathbb{R} \rightarrow \mathcal{K}_{\mathcal{P}}$, the lines $\omega \times \mathbb{R}$ map onto the composants of $\mathcal{K}_{\mathcal{P}}$, which are all immersed $\mathbb{R}$ 's except for the composants of $\mu(e)$ and $\mu\left(e^{*}\right)$ which are immersed half-lines $[0, \infty)$. We shall use the immersion $i_{\mathcal{S}_{\mathcal{P}}}: \mathbb{R} \rightarrow \mathcal{S}_{\mathcal{P}}, i_{\mathcal{S}_{\mathcal{P}}}(t)=\pi(e, t)$, and the immersion $i_{\mathcal{K}_{\mathcal{P}}}:[0, \infty) \rightarrow$ $\mathcal{K}_{\mathcal{P}}, i_{\mathcal{K}_{\mathcal{P}}}=\left.\mu \circ i_{\mathcal{S}_{\mathcal{P}}}\right|_{[0, \infty)}$.

Proof of Proposition 9.3. Fix a homeomorphism $\widehat{f}: \mathcal{K}_{\mathcal{P}} \rightarrow \mathcal{K}_{\mathcal{P}}$. We may assume that $\widehat{f}$ fixes $\mu(e)$; otherwise $\widehat{s} \circ \widehat{f}$ fixes $\mu(e)$, and if $f$ is its lift then $s_{e}^{*} \circ f$ is the lift of $\widehat{f}$. We have a homeomorphism $\nu:[0, \infty) \rightarrow[0, \infty)$ given by $\nu:=$ $i_{\mathcal{K}_{\mathcal{P}}}^{-1} \circ \widehat{f} \circ i_{\mathcal{S}_{\mathcal{P}}}$, which we use to define a map $f_{0}: i_{\mathcal{S}_{\mathcal{P}}}([0, \infty)) \rightarrow i_{\mathcal{S}_{\mathcal{P}}}([0, \infty))$ on a dense subset of $\mathcal{S}_{\mathcal{P}}$ by $f_{0}\left(i_{\mathcal{S}_{\mathcal{P}}}(t)\right)=i_{\mathcal{S}_{\mathcal{P}}} \circ \nu(t), t \geq 0$. We claim that $f_{0}$ is uniformly continuous so that it uniquely extends to a continuous map $f_{1}: \mathcal{S}_{\mathcal{P}} \rightarrow \mathcal{S}_{\mathcal{P}}$. Note that then $f_{1}$ is a lift of $\widehat{f}$ and that any such lift that preserves the orientation of the composant through $e$ must coincide with $f_{1}$. Also $f_{1}$ must be a homeomorphism: an analogous reasoning applied to $f^{-1}$ yields the inverse of $f_{1}$.

To prove the claim, fix $\varepsilon>0$ such that $\varepsilon / 2<\varrho$ where $\varrho$ is as in Lemma 9.5. There is $\delta>0$ such that $d_{\mathcal{K}_{\mathcal{P}}}(\widehat{f}(u), \widehat{f}(v))<\varepsilon / 8$ when $d_{\mathcal{K}_{\mathcal{P}}}(u, v)<\delta$, $u, v \in \mathcal{K}_{\mathcal{P}}$. Suppose that $d_{\mathcal{S}_{\mathcal{P}}}\left(i_{\mathcal{S}_{\mathcal{P}}}\left(x_{1}\right), i_{\mathcal{S}_{\mathcal{P}}}\left(x_{2}\right)\right)<\delta$. We shall show that $d_{\mathcal{S}_{\mathcal{P}}}\left(f_{0} \circ i_{\mathcal{S}_{\mathcal{P}}}\left(x_{1}\right), f_{0} \circ i_{\mathcal{S}_{\mathcal{P}}}\left(x_{2}\right)\right)<\varepsilon$. Because translations are isometries of $\mathcal{S}_{\mathcal{P}}$, we have $d_{\mathcal{S}_{\mathcal{P}}}\left(i_{\mathcal{S}_{\mathcal{P}}}\left(x_{1}+t\right), i_{\mathcal{S}_{\mathcal{P}}}\left(x_{2}+t\right)\right)<\delta$; hence also $d_{\mathcal{K}_{\mathcal{P}}}\left(i_{\mathcal{K}_{\mathcal{P}}}\left(x_{1}+t\right)\right.$, $\left.i_{\mathcal{K}_{\mathcal{P}}}\left(x_{2}+t\right)\right)<\delta$ for all $t \geq 0$. Since we assumed that $\widehat{f}(\mu(e))=\mu(e)$, the composant of $e$ is mapped onto itself, so (cf. Fact 5.3)

$$
\widehat{f} \circ i_{\mathcal{K}_{\mathcal{P}}}\left(x_{i}+t\right)=i_{\mathcal{K}_{\mathcal{P}}}\left(y_{i}+\tau_{i}(t)\right)
$$


where $y_{i}=\nu\left(x_{i}\right)$ and $\tau_{i}:[0, \infty) \rightarrow[0, \infty)$ is a homeomorphism, $i=1,2$. By the choice of $\delta$,

$$
d_{\mathcal{K}_{\mathcal{P}}}\left(i_{\mathcal{K}_{\mathcal{P}}}\left(y_{1}+\tau_{1}(t)\right), i_{\mathcal{K}_{\mathcal{P}}}\left(y_{2}+\tau_{2}(t)\right)\right)<\varepsilon / 8, \quad t \geq 0 .
$$

If $d_{\mathcal{S}_{\mathcal{P}}}\left(i_{\mathcal{S}_{\mathcal{P}}}\left(y_{1}\right), i_{\mathcal{S}_{\mathcal{P}}}\left(y_{2}\right)\right)<\varepsilon / 8$, we are done. Otherwise, $d_{\mathcal{S}_{\mathcal{P}}}\left(i_{\mathcal{S}_{\mathcal{P}}}\left(y_{1}\right)\right.$, $\left.i_{\mathcal{S}_{\mathcal{P}}}\left(-y_{2}\right)\right)<\varepsilon / 8$ from the definition of $d_{\mathcal{K}_{\mathcal{P}}}$ and (9.1) at $t=0$. Therefore, $d_{\mathcal{S}_{\mathcal{P}}}\left(i_{\mathcal{S}_{\mathcal{P}}}\left(y_{1}+t\right), i_{\mathcal{S}_{\mathcal{P}}}\left(-y_{2}+t\right)\right)<\varepsilon / 8$ and hence $d_{\mathcal{K}_{\mathcal{P}}}\left(\mu \circ i_{\mathcal{S}_{\mathcal{P}}}\left(y_{1}+t\right)\right.$, $\left.\mu \circ i_{\mathcal{S}_{\mathcal{P}}}\left(-y_{2}+t\right)\right)<\varepsilon / 8$, for all $t \geq 0$. Together with (9.1), this yields

$$
d_{\mathcal{K}_{\mathcal{P}}}\left(\mu \circ i_{\mathcal{S}_{\mathcal{P}}}\left(y_{2}+\tau(t)\right), \mu \circ i_{\mathcal{S}_{\mathcal{P}}}\left(-y_{2}+t\right)\right)<\varepsilon / 8+\varepsilon / 8=\varepsilon / 4
$$

where $\tau:=\tau_{2} \circ \tau_{1}^{-1}$ and $t \geq 0$. From Lemma $9.5, d_{\mathcal{S}_{\mathcal{P}}}\left(i_{\mathcal{S}_{\mathcal{P}}}\left(y_{2}\right), i_{\mathcal{S}_{\mathcal{P}}}\left(-y_{2}\right)\right)<$ $2 \varepsilon / 4=\varepsilon / 2$, and so $d_{\mathcal{S}_{\mathcal{P}}}\left(i_{\mathcal{S}_{\mathcal{P}}}\left(y_{1}\right), i_{\mathcal{S}_{\mathcal{P}}}\left(y_{2}\right)\right)<2 \varepsilon / 2=\varepsilon$.

Proof of Theorem 4. (i) By Proposition 9.3, $\widehat{f}$ lifts to a homeomorphism $f: \mathcal{S}_{\mathcal{P}} \rightarrow \mathcal{S}_{\mathcal{P}}$. As before, we may suppose that $\widehat{f}(\mu(e))=\mu(e)$ and $f(e)=$ $e$, as otherwise one can consider $\widehat{s} \circ \widehat{f}$. Corollary 6.1 supplies a lift $F$ : $\Lambda_{\mathcal{P}} \times \mathbb{R} \rightarrow \Lambda_{\mathcal{P}} \times \mathbb{R}$ of $f$, and a suitable adjustment by a deck transformation assures that $F(e, 0)=(e, 0)$, i.e. $\sigma=e$ in (6.1). We define a homotopy $F_{t}: \Lambda_{\mathcal{P}} \times \mathbb{R} \rightarrow \Lambda_{\mathcal{P}} \times \mathbb{R}, t \in[0,1]$, by

$$
F_{t}(\omega, x)=\left(\phi_{a / b}(\omega), \frac{a\left(x+I_{b}(\omega)\right)}{b}+t \cdot \delta(\omega, x)\right)
$$

where $a, b$ are co-prime $\mathcal{P}$-recurrent and $\delta$ is continuous and equivariant, $\delta \circ D=\delta$. We claim that $F_{t}$ factors through $\mu \circ \pi$ to a homotopy $\widehat{f}_{t}: \mathcal{K}_{\mathcal{P}} \rightarrow$ $\mathcal{K}_{\mathcal{P}}$ which connects $\widehat{f}$ to the standard map $\widehat{g}_{a / b,+}$. To prove the claim, we have to show that there is $\kappa: \Lambda_{\mathcal{P}} \rightarrow \mathbb{Z}$ such that

$$
F_{t} \circ R=D^{\kappa(\cdot)} \circ R \circ F_{t}
$$

for all $t \in[0,1]$. The above equation, explicitly written, amounts to the following two identities imposed for all $(\omega, t) \in \Lambda_{\mathcal{P}} \times \mathbb{R}$ :

$$
\phi_{a / b}\left(\omega^{-1}\right)=\gamma^{k(\omega)} \cdot\left(\phi_{a / b}(\omega)\right)^{-1}
$$

and

$$
\begin{aligned}
(a / b) \cdot I_{b}\left(\omega^{-1},-x\right)+(a / b) \cdot I_{b}(\omega, x) & +k(\omega) \\
& =-t \cdot\left(\delta\left(\omega^{-1},-x\right)+\delta(\omega, x)\right) .
\end{aligned}
$$

Both $F_{0}$ and $F_{1}$ factor to a map of $\mathcal{K}_{\mathcal{P}}: F_{0}$ covers $\widehat{g}_{a / b,+}$ and $F_{1}$ covers $\widehat{f}$. Thus (9.2) is satisfied for $t=0,1$, with the same $\kappa$ determined uniquely by (9.3). Moreover, inspection of (9.4) at $t=0$ and $t=1$ yields $\delta \circ R=-\delta$. It follows that (9.4), and thus (9.2), holds for all $t \in[0,1]$.

(ii) Let $F_{0}$ and $F_{1}$ be as in the proof of (i) above. Suppose that $H$ : $\Lambda_{\mathcal{P}} \times \mathbb{R} \rightarrow \Lambda_{\mathcal{P}} \times \mathbb{R}$ is the semi-conjugacy, $H \circ F_{1}=H \circ F_{0}$ between $F_{1}$ and $F_{0}$ obtained via global shadowing as in Proposition 8.2. From (9.2), if $(\omega, y)$ 
shadows $(\omega, x)$ then $R(\omega, y)$ shadows $R(\omega, x)$, i.e. $H \circ R=R \circ H$. It follows that $H$ descends to a semi-conjugacy $h: \mathcal{K}_{\mathcal{P}} \rightarrow \mathcal{K}_{\mathcal{P}}$ such that $h \circ \widehat{f}_{1}=h \circ \widehat{f}_{0}$.

(iii) Because $\mu$ is finite-to-one, the entropy of $\widehat{f}$ coincides with that of its lift $f: \mathcal{S}_{\mathcal{P}} \rightarrow \mathcal{S}_{\mathcal{P}}$ by Theorem 17 of [4] (cf. Section 10). Theorem 2 completes the proof.

10. Appendix: Bowen's Theorem. The following result generalizes Theorem 17 of [4], which can be obtained as the special case when $X=K$ is compact and $G$ is a trivial group.

THEOREM 5. Suppose the following hypotheses are satisfied:

(i) $(X, d)$ and $(Y, e)$ are metric spaces, $Y$ is compact, and $\pi: X \rightarrow Y$ is continuous and surjective;

(ii) a group $G$ acts on $X$ and $Y$ by isometries (on the left), and $g \circ \pi=$ $\pi \circ g$ for all $g \in G$;

(iii) $K \subset X$ is a compact set intersecting every orbit of $G$ and there are $\eta, b>0$ with $\#\{g \in G: g(A) \cap K \neq \emptyset\} \leq b$ for all $A \subset X$ with $\operatorname{diam}(A) \leq \eta$;

(iv) $T: X \rightarrow X$ and $S: Y \rightarrow Y$ are continuous and $\pi \circ T=S \circ \pi$;

(v) for every $g \in G$ there exists $h \in G$ such that $T \circ g=h \circ T$.

Then we have the following inequality for topological entropies:

$$
h(T, K) \leq h(S)+\sup _{y \in Y} h\left(T, \pi^{-1}(y) \cap K\right) .
$$

In applications, the natural projection $\pi_{G}: X \rightarrow X / G$ is typically a covering onto a compact Hausdorff space, and then (iii) is satisfied by any compact $K \subset X$ with $\pi_{G}(K)=X / G$. For $t: X / G \rightarrow X / G$ obtained as the quotient of $T$ by $G,(10.1)$ then yields

$$
h(t) \leq h(S)+\sup _{y \in Y} h\left(T, \pi^{-1}(y)\right)
$$

because $h\left(T, \pi^{-1}(y) \cap K\right) \leq h\left(T, \pi^{-1}(y)\right)$ and $h(t) \leq h(T, K)$. (In fact, $h(t)=h(T, K)$.)

ExAmple. Fix $\omega, \gamma \in \mathbb{R}$ with $\gamma$ irrational and let $\phi$ be a $\mathbb{Z}^{2}$-periodic function on $\mathbb{R}^{2}$, e.g. $\phi\left(x_{1}, x_{2}\right)=\frac{1}{4}\left(\sin \left(2 \pi x_{1}\right)+\sin \left(2 \pi x_{2}\right)+5\right)$. Let $X=$ $\mathbb{R} / \mathbb{Z} \times \mathbb{R}$ and $T\left(x_{1}, x_{2}\right):=\left(x_{1}, x_{2}\right)+(\omega, 0)+\phi\left(x_{1}, x_{2}\right)(1, \gamma)$ where $x_{1}$ is taken $\bmod 1$. Set $Y=\mathbb{R} / \mathbb{Z}$ and $S\left(x_{1}\right)=x_{1}+\omega$ so that $\pi \circ T=S \circ \pi$ for $\pi\left(x_{1}, x_{2}\right)=x_{1}-x_{2} / \gamma$. Finally, let the action of $G=\mathbb{Z}$ on $X$ be generated by $\left(x_{1}, x_{2}\right) \mapsto\left(x_{1}, x_{2}+1\right)$ and take $K=\mathbb{R} / \mathbb{Z} \times[0,1]$. Taking the quotient of $T$ by $G$ yields $t: \mathbb{R}^{2} / \mathbb{Z}^{2} \rightarrow \mathbb{R}^{2} / \mathbb{Z}^{2}$ that is a torus map homotopic to the identity that permutes leaves of a dense foliation. If $t$ is a homeomorphism, then $h\left(T, \pi^{-1}(y)\right)=0$ (cf. Lemma 7.3), and (10.2) implies that $h(t)=0$. 
QUESTION. Is the rotation set of $t$ a single point in the case when $t$ is a homeomorphism and $\omega, \gamma$, and 1 are rationally independent?

In the rationally dependent case, the answer can be extracted from [13].

Proof of Theorem 5. The argument is an extension of that of Bowen and we use many definitions and notations from [4]. In particular, $e_{n}(x, y)$ $:=\max _{i=0}^{n-1} e\left(S^{i}(x), S^{i}(y)\right)$ and $B_{n}(z, r, T):=\left\{x \in X: d\left(T^{i}(x), T^{i}(z)\right)<r\right.$, $0 \leq i<n\}$.

Set $a:=\sup _{y \in Y} h\left(T, \pi^{-1}(y) \cap K\right)$. Let $\alpha, M>0$ be arbitrary and let $\varepsilon>0$ be arbitrary yet small enough so that $d(T(x), T(\widetilde{x})) \leq \eta / 2$ if $d(x, \widetilde{x})<2 \varepsilon$. For each $y \in Y$, pick an $(m(y), \varepsilon)$-spanning set $E_{y} \subset X$ for $\pi^{-1}(y) \cap K$ so that

$$
m(y) \geq M, \quad a+\alpha \geq h\left(T, \pi^{-1}(y) \cap K\right) \geq \frac{1}{m(y)} \log \# E_{y} .
$$

Set $U_{y}:=\bigcup_{z \in E_{y}} B_{m(y)}(z, 2 \varepsilon, T)$. Observe that $\pi^{-1}(y) \cap K \subset U_{y}$; and compactness of $K$ assures that

$$
\exists_{\gamma=\gamma(y)>0} \quad \pi^{-1}(B(y, \gamma)) \cap K \subset U_{y},
$$

where $B(y, \gamma)$ is the ball of radius $\gamma$ about $y$. Fix $y_{1}, \ldots, y_{r}$ so that $\left\{B\left(y_{i}, \gamma\left(y_{i}\right)\right)\right\}_{i=1}^{r}$ is a covering of $Y$, and let $\delta>0$ be the Lebesgue number of this covering.

Consider an arbitrary $n \in \mathbb{N}$, and let $E_{n}$ be an $(n, \delta)$-spanning set for $Y$. Fix $x \in E_{n}$. Consider also an arbitrary $(n, 4 \varepsilon)$-separated subset $F \subset X \cap K$. We estimate the cardinality of the set $F_{x}:=\left\{p \in F: e_{n}(\pi(p), x) \leq \delta\right\}$. To this end, we assign to each $p \in F_{x}$ a sequence $(\mathbf{g}, \mathbf{z})=\left(g_{0}, \ldots, g_{q} ; z_{0}, \ldots, z_{q}\right)$ where $q=q(p)$ depends on $p, g_{j} \in G$, and $z_{i_{j}} \in E_{y_{i_{j}}}$ for some $i_{j} \in\{1, \ldots, r\}$, $j=0, \ldots, q$. We refer to $(\mathbf{g}, \mathbf{z})$ as the code of $p$. Fix $p \in F_{x}$. We proceed recursively.

SteP 0: Define $t_{0}:=0, p_{0}:=p$, and $x_{0}:=x$. Set $g_{0}=\mathbf{e}$ where $\mathbf{e}$ is the neutral element of $G$, and select $i_{0}$ so that $\bar{B}(x, \delta) \subset B\left(y_{i_{0}}, \gamma\left(y_{i_{0}}\right)\right)$. By (10.4), we can pick $z_{0} \in E_{y_{i_{0}}}$ with

$$
d_{m\left(y_{i_{0}}\right)}\left(z_{0}, p_{0}\right)<2 \varepsilon .
$$

STEP $s+1$ : Suppose that $\left(g_{0}, \ldots, g_{s} ; z_{0}, \ldots, z_{s}\right)$ are already defined, and so are $\left(i_{0}, \ldots, i_{s}\right)$. If

$$
t_{s+1}:=t_{s}+m\left(y_{i_{s}}\right)=m\left(y_{i_{0}}\right)+\ldots+m\left(y_{i_{s}}\right) \geq n,
$$

we stop, set $q=s$, and $(\mathbf{g}, \mathbf{z})=\left(g_{0}, \ldots, g_{q} ; z_{0}, \ldots, z_{q}\right)$ is the code of $p$. Otherwise, when $t_{s+1}<n$, we define $p_{s+1}=T^{m\left(y_{i_{s}}\right)}\left(p_{s}\right)=T^{t_{s+1}}(p), x_{s+1}=$ $S^{m\left(y_{i_{s}}\right)}\left(x_{s}\right)=S^{t_{s+1}}(x)$, and proceed as follows. Pick $g_{s+1} \in G$ so that $g_{s+1}\left(p_{s+1}\right) \in K$. Select $i_{s+1}$ so that $\bar{B}\left(g_{s+1}\left(x_{s+1}\right), \delta\right) \subset B\left(y_{i_{s+1}}, \gamma\left(y_{i_{s+1}}\right)\right)$. 
From $e_{n}(\pi(p), x) \leq \delta, e\left(S^{t_{s+1}} \circ \pi(p), S^{t_{s+1}}(x)\right) \leq \delta$; and

$$
\begin{aligned}
e\left(S^{t_{s+1}} \circ \pi(p), S^{t_{s+1}}(x)\right) & =e\left(g_{s+1} \circ S^{t_{s+1}} \circ \pi(p), g_{s+1} \circ S^{t_{s+1}}(x)\right) \\
& =e\left(g_{s+1} \circ \pi \circ T^{t_{s+1}}(p), g_{s+1}\left(x_{s+1}\right)\right) \\
& =e\left(\pi \circ g_{s+1}\left(p_{s+1}\right), g_{s+1}\left(x_{s+1}\right)\right)
\end{aligned}
$$

by hypotheses (ii) and (iv). Therefore,

$$
e\left(\pi \circ g_{s+1}\left(p_{s+1}\right), g_{s+1}\left(x_{s+1}\right)\right) \leq \delta .
$$

By (10.7), the choice of $i_{s+1}$, and (10.4), we can pick $z_{s+1} \in E_{y_{i_{s+1}}}$ with

$$
d_{m\left(y_{i s+1}\right)}\left(z_{s+1}, g_{s+1}\left(p_{s+1}\right)\right)<2 \varepsilon \text {. }
$$

This ends the iteration step.

Claim 1. If $p, \widetilde{p} \in F_{x}$ have the same code $(\mathbf{g}, \mathbf{z})=\left(g_{0}, \ldots, g_{q} ; z_{0}, \ldots, z_{q}\right)$, then $p=\widetilde{p}$.

Indeed, for $s=0, \ldots, q$, we have $d_{m\left(y_{i_{s}}\right)}\left(z_{s}, g_{s}\left(p_{s}\right)\right)<2 \varepsilon$ (cf. (10.5) and (10.8)), that is, $d\left(T^{k}\left(z_{s}\right), T^{k} \circ g_{s}\left(p_{s}\right)\right)<2 \varepsilon$ for $k=0, \ldots, m\left(y_{i_{s}}\right)-1$. Hypothesis (v) supplies $h_{s, k} \in G, 0 \leq s \leq q, 0 \leq k<m\left(y_{i_{s}}\right)$, with $T^{k} \circ g_{s}=$ $h_{s, k} \circ T^{k} ;$ and we obtain

$$
d\left(h_{s, k}^{-1} \circ T^{k}\left(z_{s}\right), T^{k}\left(p_{s}\right)\right)=d\left(T^{k}\left(z_{s}\right), h_{s, k} \circ T^{k}\left(p_{s}\right)\right)<2 \varepsilon,
$$

since $G$ acts by isometries (hypothesis (ii)). From (10.9) and its analogue for $\widetilde{p}, d\left(T^{i}(p), T^{i}(\widetilde{p})\right)<4 \varepsilon$ for $i=0, \ldots, t_{q+1}-1$. Since $t_{q+1}-1 \geq n-1$ by (10.6), and $p, \widetilde{p} \in F$, we must have $p=\widetilde{p}$, which proves the claim.

Set $\mu:=\max _{i=1}^{r} m\left(y_{i}\right)$.

Claim 2. \#\{(g, $\mathbf{z}):(\mathbf{g}, \mathbf{z})$ is a code of some $\left.p \in F_{x}\right\} \leq b^{n / M} e^{(a+\alpha)(n+\mu)}$.

Note that the two claims put together imply $\# F \leq \sum_{x \in E_{n}} \# F_{x} \leq$ $\# E_{n} \cdot b^{n / M} e^{(a+\alpha)(n+\mu)}$. Since $n, \varepsilon, F$ and $E_{n}$ were arbitrary, we conclude that $h(T, K) \leq h(S)+(\log b) / M+(a+\alpha)$ so that (10.1) follows from arbitrariness of $M, \alpha>0$.

It remains to demonstrate Claim 2. To ease the exposition, we build a weighted graph as follows. For vertices we take all $(\mathbf{g}, \mathbf{z})=\left(g_{0}, \ldots, g_{s}\right.$; $\left.z_{0}, \ldots, z_{s}\right)$ that are initial segments of a code, i.e. $\left(g_{0}, \ldots, g_{q} ; z_{0}, \ldots, z_{q}\right)$ with $q \geq s$ is a code of some $p \in F_{x}$ for certain $g_{s+1}, \ldots, g_{q}$ and $z_{s+1}, \ldots, z_{q}$. We also attach to each such vertex a weight equal to $m\left(y_{i_{s}}\right)$ and call $s$ the level of the vertex. Then we place a directed edge from $(\mathbf{g}, \mathbf{z})=\left(g_{0}, \ldots, g_{s}\right.$; $\left.z_{0}, \ldots, z_{s}\right)$ to $(\widetilde{\mathbf{g}}, \widetilde{\mathbf{z}})=\left(\widetilde{g}_{0}, \ldots, \widetilde{g}_{s+1} ; \widetilde{z}_{0}, \ldots, \widetilde{z}_{s+1}\right)$ iff $g_{i}=\widetilde{g}_{i}$ and $z_{i}=\widetilde{z}_{i}$ for $0 \leq i \leq s$; and we say that $(\widetilde{\mathbf{g}}, \widetilde{\mathbf{z}})$ follows $(\mathbf{g}, \mathbf{z})$. The resulting graph $\mathcal{G}$ is clearly a collection of directed trees.

Claim 3. Fix a vertex $(\mathbf{g}, \mathbf{z})=\left(g_{0}, \ldots, g_{s} ; z_{0}, \ldots, z_{s}\right)$ and consider the set $V$ of all vertices $(\widetilde{\mathbf{g}}, \widetilde{\mathbf{z}})=\left(g_{0}, \ldots, g_{s+1} ; z_{0}, \ldots, z_{s+1}\right)$ following $(\mathbf{g}, \mathbf{z})$. 
There are at most $b$ different $g_{s+1} \in G$ that appear in $V$. Moreover, for $g \in G$, all vertices in $V_{g}:=\left\{(\widetilde{\mathbf{g}}, \widetilde{\mathbf{z}}) \in V: g_{s+1}=g\right\}$ have the same weight equal to some $m$ and $\# V_{g} \leq e^{(a+\alpha) m}$.

For the proof, let $B$ collect all $p \in F_{x}$ with an initial segment of their code coinciding with $(\mathbf{g}, \mathbf{z})$. Setting $k=m\left(y_{i_{s}}\right)-1$ in (10.9) yields $d\left(h_{s, k}^{-1} \circ\right.$ $\left.T^{k}\left(z_{s}\right), T^{t_{s+1}-1}(p)\right)<2 \varepsilon$ for all $p \in B$. By the choice of $\varepsilon, d\left(T \circ h_{s, k}^{-1} \circ\right.$ $\left.T^{k}\left(z_{s}\right), T^{t_{s+1}}(p)\right)<\eta / 2$ for $p \in B$ so that $\operatorname{diam}(A) \leq \eta$ for $A:=T^{t_{s+1}}(B)$. Any $g_{s+1}$ appearing in $V$ has $p \in B$ with $g_{s+1}\left(p_{s+1}\right) \in K$ so that $g_{s+1}(A) \cap$ $K \neq \emptyset$. Hypothesis (iii) allows then for at most $b$ possibilities for $g_{s+1}$. Once $g_{s+1}$ is fixed so is $i_{s+1}$ and there are at most $\# E_{y_{i_{s+1}}} \leq e^{(a+\alpha) m\left(y_{i_{s+1}}\right)}$ possibilities for $z_{s+1} \in E_{y_{i_{s+1}}}$. Also, all the vertices in $V_{g_{s+1}}$ have the same weight $m\left(y_{i_{s+1}}\right)$. This ends the proof of Claim 3 .

Each code $(\mathbf{g}, \mathbf{z})=\left(g_{0}, \ldots, g_{q} ; z_{0}, \ldots, z_{q}\right)$ of $p \in F_{x}$ determines a unique maximal path in $\mathcal{G}$ starting at some root (a level-0 vertex). The weight of that path is $t_{q+1}=m\left(y_{i_{0}}\right)+\ldots+m\left(y_{i_{q}}\right)=t_{q}+m\left(y_{i_{q}}\right)<n+\mu$. Claim 2 is then a consequence of Claim 3 and the following general lemma.

Lemma 10.6. Suppose that $\mathcal{G}$ is a collection of trees with weighted vertices and there are $b, M, A>0$ so that

(a) no vertex is lighter than $M$;

(b) there is $m_{0}$ so that there are at most $e^{A m_{0}}$ roots and each root is heavier than $m_{0}$;

(c) for any vertex, all vertices following it can be grouped into sets $U_{1}, \ldots, U_{\widetilde{b}}, \widetilde{b} \leq b$, so that $\# U_{i} \leq e^{A m_{i}}$ and each vertex in $U_{i}$ is heavier than $m_{i}$ for some $m_{i} \geq 0, i=1, \ldots, \widetilde{b}$.

If no path in $\mathcal{G}$ is heavier than $N$, then the number of different maximal paths (starting at a root) does not exceed $b^{N / M} e^{A N}$.

Proof. We proceed by induction on the height $h$ defined as the maximal level of a vertex in $\mathcal{G}$. If $h=0$, then $\mathcal{G}$ has only roots; their number does not exceed $e^{A m_{0}}$ where $N \geq m_{0}$ by (b). If $h>0$, for any fixed root $R$ that is followed by some level- 1 vertices, we group those vertices into $U_{1}, \ldots, U_{\widetilde{b}}$, $\widetilde{b} \leq b$, as stipulated by hypothesis (c). For every maximal path $P$ in $\mathcal{G}$ starting at $R$, there is a unique $i \in\{1, \ldots, \widetilde{b}\}$ so that $P$ passes through some $W \in U_{i}$; let $Q$ be the maximal subpath of $P$ that starts at $W$. There are at most $b^{(N-M) / M} e^{A\left(N-m_{0}\right)}$ possibilities for $Q$ for any fixed $i$ since, by (c), the induction hypothesis applies to the collection $\mathcal{G}_{i}$ of the maximal subtrees of $\mathcal{G}$ that are rooted at the vertices in $U_{i}$. By summing over all roots and all $i$, we see that the number of possibilities for $P$ cannot exceed

$$
e^{A m_{0}} \cdot b \cdot b^{N /(N-M)} e^{A\left(n-m_{0}\right)}=b^{n / M} e^{A n} .
$$




\section{References}

[1] J. M. Aarts and J. Fokkink, The classification of solenoids, Proc. Amer. Math. Soc. 111 (1991), 1161-1163.

[2] L. M. Abramov, The entropy of an automorphism of a solenoidal group, Teor. Veroyatnost. i Primenen. 4 (1959), 249-254 (in Russian).

[3] M. Barge, The topological entropy of homeomorphisms of Knaster continua, Houston J. Math. 13 (1987), 465-485.

[4] R. Bowen, Entropy for group endomorphisms and homogeneous spaces, Trans. Amer. Math. Soc. 153 (1971), 401-414.

[5] D. van Dantzig, Über topologisch homogene Kontinua, Fund. Math. 15 (1930), 102125.

[6] W. Dębski, The positions of composants in indecomposable arc-like continua of the simplest type, preprint.

[7] R. Fokkink, The structure of trajectories, PhD thesis, Technische Univ. Delft, 1991.

[8] J. Franks, Anosov diffeomorphisms on tori, Trans. Amer. Math. Soc. 145 (1969), $117-124$.

[9] M. Handel, Global shadowing of pseudo-Anosov homeomorphisms, Ergodic Theory Dynam. Systems 5 (1985), 373-377.

[10] E. Hewitt and K. A. Ross, Abstract Harmonic Analysis, I, Springer, 1963.

[11] A. Katok and B. Hasselblatt, Introduction to the Modern Theory of Dynamical Systems, Cambridge Univ. Press, 1995.

[12] J. Keesling, The group of homeomorphisms of a solenoid, Trans. Amer. Math. Soc. 172 (1972), 119-131.

[13] J. Kwapisz, The Poincaré rotation number for maps of the real line with almost periodic displacement, Nonlinearity 13 (2000), 1841-1854.

[14] D. Lind and T. Ward, Automorphisms of solenoids and p-adic entropy, Ergodic Theory Dynam. Systems 8 (1988), 411-419.

[15] M. C. McCord, Inverse limit sequences with covering maps, Trans. Amer. Math. Soc. 114 (1965), 197-209.

[16] P. Minc, Solenoids and bihomogeneity, in: Continua with the Houston Problem Book, Lecture Notes in Pure Appl. Math. 170, Dekker, New York, 1995, 297-304.

[17] M. W. Mislove and J. T. Rogers, Local product structures on homogeneous continua, Topology Appl. 31 (1989), 259-267.

[18] W. Scheffer, Maps between topological groups that are homotopic to homomorphisms, Proc. Amer. Math. Soc. 33 (1972), 562-567.

[19] E. H. Spanier, Algebraic Topology, McGraw-Hill, New York, 1966, page 67.

[20] L. Vietoris, Über den höheren Zusammenhang kompakter Räume und eine Klasse von zusammenhangstreuen Abbildungen, Math. Ann. 97 (1927), 454-472.

[21] P. Walters, An Introduction to Ergodic Theory, Grad. Texts in Math. 79, Springer, New York, 1982.

Department of Mathematical Sciences

Montana State University

Bozeman, MT 59717-2400, U.S.A.

E-mail: jarek@math.montana.edu

Web: http://www.math.montana.edu/ jarek/

Received 15 March 2000;

in revised form 28 February 2001 